\title{
OPEN PROJECTIONS IN OPERATOR ALGEBRAS I: COMPARISON THEORY
}

\author{
DAVID P. BLECHER AND MATTHEW NEAL
}

\begin{abstract}
We begin a program of generalizing basic elements of the theory of comparison, equivalence, and subequivalence, of elements in $C^{*}$-algebras, to the setting of more general algebras. In particular, we follow the recent lead of Lin, Ortega, Rørdam, and Thiel of studying these equivalences, etc., in terms of open projections or module isomorphisms. We also define and characterize a new class of inner ideals in operator algebras, and develop a matching theory of open partial isometries in operator ideals which simultaneously generalize the open projections in operator algebras (in the sense of the authors and Hay), and the open partial isometries (tripotents) introduced by the authors.
\end{abstract}

\section{IntRoduction AND NOTATION}

Inspired by a recent paper of Ortega, Rørdam, and Thiel [31], we begin a program of generalizing basic elements of the theory of comparison, equivalence, and subequivalence, of elements in $C^{*}$-algebras, to the setting of more general algebras. To do this we establish several technical results and tools, such as a new class of inner ideals in operator algebras, and a matching theory of 'open partial isometries' which simultaneously generalize the open projections in operator algebras (in the sense of the authors and Hay [9]), and (essentially) the open partial isometries or tripotents introduced in 12 .

We begin by considering a relation between two elements $a$ and $b$ which one may define in any monoid or algebra $A$ : namely that there exists $x, y \in A$ with $a=x y, b=y x$. If $A$ is a group, then this defines an equivalence relation. In an algebra this is not an equivalence relation in general. In fact this fails to be an equivalence relation even for the case $A=M_{n}$, the $n$ by $n$ matrices. Indeed even in this simple case the characterization of when two matrices $a$ and $b$ can be related in this way is quite subtle [27, and does not match any kind of equivalence relation on a $C^{*}$-algebra that has been important in $C^{*}$-algebra theory as far as we know. The question arises of how to fix this problem in an operator algebra, by which we will mean a (not necessarily selfadjoint) subalgebra of $B(H)$, for a Hilbert space $H$. In a $C^{*}$-algebra $A$ the 'fix' that works, on a large subset of $A$, is to insist that $y=x^{*}$ above; and then this defines an equivalence relation $\sim$ on the positive cone $A_{+}$of $A$. This is sometimes called Pedersen equivalence (see e.g. 33]). We will

2010 Mathematics Subject Classification. Primary 46L85, 46H10, 46L07, 47L30; Secondary 06F25, 17C65, 46L08, 47L07.

Key words and phrases. TRO's, JB*-triples, Hilbert $\mathrm{C}^{*}$-modules, nonselfadjoint operator algebra, open projection, Cuntz semigroup, comparison theory, equivalence relations on an operator algebra, hereditary subalgebra, ideals, partial isometry.

February 7, 2012. 
expand this 'fix' to a larger set than $A_{+}$, and to more general operator algebras than $C^{*}$-algebras.

In $C^{*}$-algebra theory, coarser equivalence relations than Pedersen equivalence, and matching notions of subequivalence or comparison, are becoming increasingly important [6]. For example, recently the study of Cuntz equivalence and subequivalence has become one of the most important areas of $C^{*}$-algebra theory (see e.g. 4, 31). And comparison theory in $C^{*}$-algebras, where one considers coarser notions of ordering of elements in a $C^{*}$-algebra than the usual $\leq$, generalizing in some sense the crucially important comparison theory of projections in a von Neumann algebra (which is often most effectively done using traces), is obviously important even in its own right. Studying these equivalence relations and comparisons involves marshaling a formidable array of tools, results, and perspectives. Some of this is simply impossible for more general operator algebras, due for example to scarcity of projections and hereditary subalgebras in general. A classification program along these lines would be misguided. Our goal in this project is simply to transfer some small portion of these tools, results, and perspectives to more general operator algebras than $C^{*}$-algebras. Along the way we were also led to develop some aspects of 'noncommutative topology and noncommutative function theory relative to an operator algebra'. In the present paper we discuss generalizations of Pedersen, Blackadar, and Peligrad-Zsidó equivalence, using the paper of Ortega, Rørdam, and Thiel 31 . as our guide. The main point is that the authors of [31 recast the equivalences and subequivalences mentioned above, and also Cuntz equivalence and subequivalence, in terms of open projections (see also [29]). In view of our generalizations of open projections in earlier projects [9, 12, 15, it was tempting to follow their approach in a more general setting.

Our variants of Pedersen, Blackadar, and Peligrad-Zsidó equivalence lead us to introduce in Section 3 the notion of a $*$-open tripotent, which may be viewed as a generalization of Akemann's open projections, and a generalization of our version of the latter for general operator algebras from 9. They also are essentially a 'non-selfadjoint variant' of the open tripotents which we introduced in [12. These *-open tripotents play a central role in our equivalence and comparison theory. One characterization of $*$-open tripotents is in terms of hereditary bimodules, which encapsulate the algebraic structure of $\overline{a A b}$ when $a$ and $b$ are Pedersen or Blackadar equivalent. We develop the theory of hereditary bimodules in Section 4. For example we show using results from [15] that every separable hereditary bimodule is of the form $\overline{a A b}$ where $a$ and $b$ are Pedersen equivalent; and that a general hereditary bimodule is the closure of an increasing union of subspaces of the same form. These sections are mostly of technical interest, for later use in the development of the theory, however applications are given there in the form of rephrasings of our variant of Pedersen equivalence in the language of $*$-open tripotents or hereditary bimodules. In Section 5 we study a variant of 'Blackadar equivalence and subequivalence' of elements in our algebra $A$, and prove the analogue of some results of Lin and Ortega-Rørdam-Thiel 29, 31. For example, elements $a$ and $b$ of the type we consider are Blackadar equivalent in our sense iff $\overline{a A} \cong \overline{b A}$ completely isometrically and as right $A$-modules. It is also equivalent to $\overline{a A b}$ being a "principal' hereditary bimodule, or to the support projections of $a$ and $b$ being equivalent in an appropriate variant of the sense of Peligrad and Zsidó. In an appendix we prove TRO versions of a few of our earlier results. 
We remark that much of our theory depends on the existence of $n$th roots of elements satisfying $\|1-a\| \leq 1$. Thus we would expect that a certain portion of this theory generalizes to bigger classes of elements which have roots, and a smaller portion may generalize further still to a bigger class of Banach algebras using the facts about roots in Banach algebras (see e.g. 28]).

Blanket convention: Throughout this paper, $A$ is a fixed operator algebra, and $B$ is a $C^{*}$-algebra that contains it. Sometimes $B$ will be generated by $A$; that is there is no proper $C^{*}$-subalgebra of $B$ containing $A$. The diagonal $\Delta(A)=\left\{a \in A: a^{*} \in A\right\}$ is a $C^{*}$-algebra and a subalgebra of $A$. Concerning notation and background, which we will discuss next, it will be helpful for the reader to have easy access to several of the references, particularly [10, 9, 12, 15, 11, for example for more detail beyond what is presented here.

For us a projection is always an orthogonal projection. We recall that by a theorem due to Ralf Meyer, every operator algebra $A$ has a unique unitization $A^{1}$ (see e.g. [10, Section 2.1]). Below 1 always refers to the identity of $A^{1}$ if $A$ has no identity. We are mostly interested in operator algebras with contractive approximate identities (cai's). We also call these approximately unital operator algebras. If $A$ is a nonunital operator algebra represented (completely) isometrically on a Hilbert space $H$ then one may identify the unitization $A^{1}$ with $A+\mathbb{C} I_{H}$. The second dual $A^{* *}$ is also an operator algebra with its (unique) Arens product, this is also the product inherited from the von Neumann algebra $B^{* *}$ if $A$ is a subalgebra of a $C^{*}$-algebra $B$. Meets and joins in $B^{* *}$ of projections in $A^{* *}$ remain in $A^{* *}$, since these meets and joins may be computed in the biggest von Neumann algebra contained inside $A^{* *}$. Note that $A$ has a cai iff $A^{* *}$ has an identity $1_{A^{* *}}$ of norm 1 , and then $A^{1}$ is sometimes identified with $A+\mathbb{C} 1_{A^{* *}}$.

For sets $X$ and $Y$ we write $X Y$ for the closure of the sum of products of the form $x y$ for $x \in X, y \in Y$, and similarly for a product of three sets. If $a \in A$ then we write $A_{a}$ for $\overline{a A a}$. An inner ideal in $A$ is a subspace $D$ with $D A D \subset D$. A hereditary subalgebra (HSA) of $A$ is an inner ideal which has a cai. For the theory of HSA's in general operator algebras see 9 . These objects are in an order preserving, bijective correspondence with the open projections $p \in A^{* *}$, by which we mean that there is a net $x_{t} \in A$ with $x_{t}=p x_{t} p \rightarrow p$ weak*. These are also the open projections $p$ in the sense of Akemann [1, 2] in $B^{* *}$, where $B$ is a $C^{*}$-algebra containing $A$, such that $p \in A^{\perp \perp}$. Indeed the weak* limit of a cai for a HSA is an open projection, and is called the support projection of the HSA. Conversely, if $p$ is an open projection in $A^{* *}$, then $p A^{* *} p \cap A$ is a HSA in $A$. We write $p A^{* *} p \cap A$ as ${ }_{p} A_{p}$, or simply as $A_{p}$. Similarly, if $q$ is another projection, then ${ }_{p} A_{q}=\{a \in A: a=p a q\}$.

We recall that a closed projection is the 'perp' of an open projection. Suprema (resp. infima) of open (resp. closed) projections in $A^{* *}$, remain in $A^{* *}$, by the fact mentioned two paragraphs earlier about meets and joins, together with the $C^{*}$-algebraic case of these facts [1, 2].

In [34, Peligrad and Zsidó introduce a notion of equivalence for open projections $p$ and $q$ in the bidual of a $C^{*}$-algebra $B$ : We say $p$ and $q$ are Peligrad-Zsidó equivalent, and write $p \sim_{\mathrm{PZ}} q$ if there is a partial isometry $v \in B^{* *}$ such that $p=v^{*} v, q=v v^{*}, v B_{p} \subset B, v^{*} B_{q} \subset B$. Peligrad and Zsidó prove in [34, Lemma 1.3] that in fact $q$ being open and $v^{*} B_{q} \subset B$ are implied by the other conditions above.

We will need some background from [12. A ternary ring of operators (or TRO for short), is a closed subspace $Z$ of a $\mathrm{C}^{*}$-algebra $A$ such that $Z Z^{*} Z \subset Z$. A 
tripotent is an element $u \in Z$ such that $u u^{*} u=u$. This is clearly simply a partial isometry in $Z$. We order tripotents by $u \leq v$ if and only if $u v^{*} u=u$. This turns out to be equivalent to $u=v u^{*} u$, or to $u=u u^{*} v$, and implies that $u^{*} u \leq v^{*} v$ and $u u^{*} \leq v v^{*}$. The linking $\mathrm{C}^{*}$-algebra $L(Z)$ of a TRO $Z$ has 'four corners' $Z Z^{*}$, $Z, Z^{*}$, and $Z^{*} Z$. Here $Z Z^{*}$ is the closure of the linear span of products $z w^{*}$ with $z, w \in Z$, and similarly for $Z^{*} Z$. The second dual of a TRO $Z$ is a TRO, which is studied in terms of the von Neumann algebra which is the second dual of $L(Z)$. An inner ideal (resp. ternary ideal) of a TRO $Z$ is defined to be a closed subspace $J$ with $J Z^{*} J \subset J$ (resp. $J Z^{*} Z \subset J$ and $Z Z^{*} J \subset J$ ).

In most of our paper, although we will be using notation and concepts from the theory of TRO's and JB*-triples, in fact the TRO concerned will simply be the $C^{*}$-algebra $B$. Thus we will write the discussion in the next few paragraphs in terms of $B$, although it all makes sense even if $B$ were a TRO. The Peirce 2-space of a tripotent $u$ in $B$ is the subset

$$
B_{2}(u)=\left\{z \in B: z=u u^{*} z u^{*} u\right\}=u u^{*} B u^{*} u .
$$

Clearly $B_{2}(u)$ is an inner ideal of $B$ in the TRO sense. There is a natural product (the Peirce product $x \cdot y=x u^{*} y$ ) and involution (namely $x^{\sharp}=u x^{*} u$ ) on $B_{2}(u)$ making the latter space into a unital $C^{*}$-algebra. The identity element is $u$. It is easy to check that $u^{*} B_{2}(u)$ is a $\mathrm{C}^{*}$-subalgebra of $B$ (this will be $B^{*} B$ if $B$ is a TRO), and the map $z \mapsto u^{*} z$ is a $*$-isomorphism from $B_{2}(u)$, with the product and involution above, onto this $\mathrm{C}^{*}$-subalgebra. We will write $B(u)$ for $B_{2}^{* *}(u) \cap B=$ $\left\{z \in B: z=u u^{*} z u^{*} u\right\}$. Here $B_{2}^{* *}(u)$ is the Peirce 2-space of $u$ in $B^{* *}$.

We will also occasionally use other facts from Sections 2 and 3 of [12, like those concerning the range tripotent $r(x)$ of an element $x \in B$. Since this is simply the partial isometry occurring in the polar decomposition of $x$, so $x=r(x)|x|$, the facts concerning range tripotents are fairly simple and essentially well known. For example, $r(x) \in B^{* *}$ and $x=\left|x^{*}\right| r(x)$.

In 12 we defined a tripotent $u$ in the second dual of a TRO to be open, if when we consider the Peirce 2-space for $u$ as a $\mathrm{W}^{*}$-algebra in the way described above, then $u$ is the weak* limit in the second dual, of an increasing net of positive elements from $B(u)$ (positive with respect to the $C^{*}$-algebra structure determined by the Pierce product; and $B(u)$ is replaced by $Z(u)$ in the case of a general TRO $Z$ rather than $B)$. Beware that this definition differs from the one given in [20]. For example, all unitaries are open in the sense of that paper. See [22, 23, 18, for a recent $\mathrm{JB}^{*}$-triple generalization of our notion.

There are several equivalent definitions of open tripotents in [12, Theorem 2.10, Corollary 3.4]. For example, a tripotent $u$ in the second dual is open iff it is a weak* limit of an increasing net of range tripotents (defined above), or if the projection

$$
\hat{u}=\frac{1}{2}\left[\begin{array}{cc}
u u^{*} & u \\
u^{*} & u^{*} u
\end{array}\right]
$$

is an open projection in Akemann's sense (discussed earlier). Another important characterization of open tripotents, as the 'support tripotent' of certain inner ideals, will be discussed in Section 4. In Proposition 6.1, we show that our open tripotents are (at least in the case that the TRO is a $C^{*}$-algebra) the partial isometries occurring in the Peligrad and Zsidó equivalence of open projections defined above. A special case of this was proven as the equivalence of (i) and (vi) in [16. In our work [12, 16] we were unaware of [34, which in retrospect clearly overlaps in small 
part with [12. Namely, as we have just seen, 34 implicitly contains the notion of an open tripotent; and a couple of the results in 34 may thus be read as results about open tripotents (these results were not repeated in [12]).

In what follows we use the notation $\mathfrak{S}_{A}$ for either $\{a \in A:\|1-2 a\| \leq 1\}$, or $\{a \in \operatorname{Ball}(A):\|1-a\| \leq 1\}$. Of course the first of these two sets is contained in the second. However the reader should choose which of these two they prefer, and stick with this choice for the rest of the paper. Note that $\mathfrak{S}_{A}$ is convex, and closed under multiplication by scalars in $[0,1]$. We write $\mathfrak{c}_{A}$ for the cone $\mathbb{R}^{+} \mathfrak{S}_{A}$. This cone will, just as in [15], play a role for us very much akin to the role of the positive cone in a $C^{*}$-algebra. Indeed an underlying philosophy in [15] and the present paper and its sequel [13] is to use this cone to generalize important facts and theories for $C^{*}$-algebras which employ the positive cone, to more general operator algebras. The set $\mathfrak{c}_{A}$ is 'large': for example in a unital operator algebra it includes $\mathbb{R}^{+}(1+\operatorname{Ball}(A))$, which spans $A$. On the other hand, if $A$ has a cai then that cai may be chosen in this cone [35], and $A=\overline{\operatorname{Span}}\left(\mathfrak{S}_{A}\right)$. To see the latter, note that by [15, Lemma 8.1] and the unital case just discussed, the weak* closure $E$ of $\overline{\operatorname{Span}}\left(\mathfrak{S}_{A}\right)$ equals $A^{* *}$, hence by the bipolar theorem $A=\overline{\operatorname{Span}}\left(\mathfrak{S}_{A}\right)$.

We will use throughout the fact from [15] that elements in $\mathfrak{S}_{A}$ have $n$th roots for all $n \in \mathbb{N}$, which are again in $\mathfrak{S}_{A}$. There is an explicit formula for these roots, and indeed for $x^{t}$ for all $0<t \leq 1$ and $\|1-x\| \leq 1$ (the series in Lemma 1.2 below). If $a \in \mathfrak{S}_{A}$, then $\left(a^{\frac{1}{n}}\right)$ converges weak ${ }^{*}$ to an open projection which is written as $p_{a}$ or $s(a)$, and this is both the left and the right support projection of $a$ (see [15, Section $2])$. If $a \in \mathfrak{c}_{A}$, its support projection $s(a)=p_{a}$ is $s\left(\frac{a}{\|a\|}\right)$. In this case $\overline{a A a}$ is a HSA of $A$, and the support projection of this HSA is $s(a)$, so $\overline{a A a}=A_{p_{a}}=p_{a} A^{* *} p_{a} \cap A$.

We will need some simple properties of the roots mentioned above:

Lemma 1.1. If $a \in \mathfrak{S}_{A}$ then $a^{r s}=\left(a^{r}\right)^{s}$ if $r, s \in(0,1]$, and $a^{r} a^{s}=a^{r+s}$ if $r, s, r+s \in(0,1]$.

Proof. This follows from the disk algebra functional calculus used to define these powers in [15.

Lemma 1.2. If $a \in \mathfrak{S}_{A}$ then a has a unique square root in $\mathfrak{S}_{A}$. Indeed for $n \in \mathbb{N}$, a has a unique nth root in $\mathfrak{S}_{A}$ whose numerical range is contained in the set of numbers re $e^{i \theta}$ with $|\theta| \leq \frac{\pi}{n}$. This nth root is $\sum_{k=0}^{\infty}\left(\begin{array}{l}t \\ k\end{array}\right)(-1)^{k}(1-a)^{k}$, where $t=\frac{1}{n}$, and this is a norm limit of polynomials in a with no constant term.

Proof. This follows from a well known operator theoretic fact concerning roots (see e.g. [28, Theorem 0.1]), together with [15, Proposition 2.3]. We remark that an early version of [15] contained this explicit formula, the published version referred to $\sum_{k=0}^{\infty} c_{k}(1-x)^{k}$ without explicitly stating the values of $c_{k}$. However that $c_{k}=$ $\left(\begin{array}{l}t \\ k\end{array}\right)(-1)^{k}$ is obvious.

Remark. A clarification, for our operators $a$, one cannot expect $\left(a^{\frac{1}{2}}\right)^{*} a^{\frac{1}{2}}=|a|$. In other words, our 'square root' $f(a)=a^{\frac{1}{2}}$ does not satisfy $f\left(a^{*} a\right)=f\left(a^{*}\right) f(a)$. To see this note that if $a$ is any invertible matrix in $\mathfrak{S}_{M_{n}}$, then $b=a^{\frac{1}{2}}$ is invertible, and so if $b^{*} b b^{*} b=a^{*} a=\left(b^{*}\right)^{2} b^{2}$ then $b b^{*}=b^{*} b$. So $b$ and hence $a$ is normal. But this is false in general.

Lemma 1.3. If $S \in B(H)$ with $\left\|I_{H}-S\right\| \leq 1$, and if $T$ is a contraction in $B(K, H)$, then $\left\|I_{K}-T^{*} S T\right\| \leq 1$. 
Proof. $I_{K}-T^{*} S T=V^{*} D V$, where $V^{*}=\left[\begin{array}{ll}\left(I_{K}-T^{*} T\right)^{\frac{1}{2}} & T\end{array}\right]$ and $D=\operatorname{diag}\left(I_{K}, I_{H}-\right.$ $S)$. Since $V$ is an isometry the result is clear.

We note that if $a \in \mathfrak{c}_{A}$ and $n \in \mathbb{N}$, then it is clear that the left and right supports of $a^{n}$ equals $s(a)$.

Lemma 1.4. If $a \in \mathfrak{S}_{A}$ and $v$ is a partial isometry in any containing $C^{*}$-algebra $B$ with $v^{*} v=s(a)$, then vav $v^{*} \in \mathfrak{S}_{B}$ and $\left(v_{a v^{*}}\right)^{r}=v a^{r} v^{*}$ if $r \in(0,1) \cup \mathbb{N}$.

Proof. This is clear if $r=k \in \mathbb{N}$. Also $v\left(v^{*} v-a\right)^{k} v^{*}=\left(v v^{*}-v a v^{*}\right)^{k}$. For $r \in(0,1)$ we have $v a^{r} v^{*}$ equal to

$$
\begin{aligned}
\sum_{k=0}^{\infty}\left(\begin{array}{l}
r \\
k
\end{array}\right)(-1)^{k} v(1-a)^{k} v^{*} & =\sum_{k=0}^{\infty}\left(\begin{array}{l}
r \\
k
\end{array}\right)(-1)^{k}\left(v v^{*}-v a v^{*}\right)^{k} \\
& =\sum_{k=0}^{\infty}\left(\begin{array}{l}
r \\
k
\end{array}\right)(-1)^{k}\left(1-v a v^{*}\right)^{k}
\end{aligned}
$$

which equals $\left(v a v^{*}\right)^{r}$, using Lemma 1.3

The following result will not be used here, but is of independent interest. It may be generalized to give the variant of [29, Lemma 3.12] appropriate to the setting of quotients of 'rigged modules' in the sense of [7].

Proposition 1.5. If $J$ is a right ideal in $A$ with a left cai $\left(e_{t}\right)$ in $\mathfrak{S}_{A}$, for example if $J=\overline{a A}$ for some $a \in \mathfrak{S}_{A}$, and if $x \in A$ then the norm of $x+J$ in $A / J$ equals $\lim _{t}\left\|\left(1-e_{t}\right) x\right\|$.

Proof. Just as in the proof of [29, Lemma 3.12].

We now consider $a, b \in \mathfrak{S}_{A}$, and are interested in $\overline{a A b}$, an inner ideal in $A$.

Proposition 1.6. If $a, b \in \mathfrak{S}_{A}$, and $p=p_{a}, q=p_{b}$, then

$$
\overline{a A b}={ }_{p} A_{q}=\{x \in A: x=p a q\}=\overline{a A} \cap \overline{A b}=\overline{a A} \overline{A b}=A_{a} A A_{b} .
$$

Proof. Clearly $a A b \subset{ }_{p} A_{q}$. On the other hand if $x \in{ }_{p} A_{q}={ }_{p} A \cap A_{q}=\overline{a A} \cap \overline{A b}$ then $a^{\frac{1}{n}} x b^{\frac{1}{n}} \rightarrow x$. So $x \in \overline{a A b}$. So

$$
\overline{a A b}={ }_{p} A_{q}=\{x \in A: x=p a q\}=\overline{a A} \cap \overline{A b} \supset \overline{a A} \overline{A b} .
$$

But of course $a A b=\left(a A b^{\frac{1}{2}}\right) b^{\frac{1}{2}} \subset \overline{a A} \overline{A b}$, and so $\overline{a A b}=\overline{a A} \overline{A b}$. Clearly $A_{a} A A_{b} \subset$ $\overline{a A b}$, and so $A_{a} A A_{b}=\overline{a A b}$ since $a \in A_{a}, b \in A_{b}$.

\section{A Pedersen type equivalence in operator algebras}

In order to fix the problem with the relation considered in the second paragraph of our paper, it is tempting to define a relation $a \sim_{c} b$ if there exist $x, y \in \operatorname{Ball}(B)$ with $a=x y, y x=b$. As we will see momentarily this is still not what one would want, but nonetheless this notation $\sim_{c}$ will be useful. Indeed $\sim_{c}$ is not an equivalence relation on $\mathfrak{S}_{A}$. However it is almost an equivalence relation in the following way: $a \sim_{c} b$ and $b \sim_{c} d$ implies that $a^{2} \sim_{c} d^{2}$. Indeed if $a=x y, y x=b=w z, d=$ $z w$ then $a^{2}=x y x y=x b y=x w z y$ and $z y x w=z b w=z w z w=d^{2}$.

The relation $\sim_{c}$ is certainly not a definition that we will consider seriously, even if one insists on extra conditions on the support projections for $x$ and $y$. Indeed even in the simple case $A=M_{2}$ this definition would be wrong in our context. 
It differs completely from our simplest equivalence relation, Pedersen equivalence, which in this case is just unitary equivalence. To see this, let $x=\operatorname{diag}\left\{\frac{1}{\sqrt{2}}, \frac{1+3 K}{\sqrt{2}}\right\}$, for some small positive $K$ to be determined, and let $y$ be the 2 by 2 matrix with rows $\frac{1+K}{\sqrt{2}}, \sqrt{2} K$ and $0, \frac{1}{\sqrt{2}}$. Then $x y$ is the 2 by 2 matrix with rows $\frac{1+K}{2}, K$ and $0, \frac{1+3 K}{2}$, whereas $y x$ is the 2 by 2 matrix with rows $\frac{1+K}{2},(1+3 K) K$ and $0, \frac{1+3 K}{2}$. For $K$ small enough both $x y$ and $y x$ are in $\mathfrak{S}_{A}$ (indeed $x$ and $y$ are in $\mathfrak{S}_{A}$ too). Simple formulae for the norm of an upper triangular 2 by 2 matrix show that $x y$ and $y x$ have different norms in general, hence are not unitarily equivalent.

The following seems to be a much more promising relation:

Definition 2.1. We say that $a$ and $b$ are root equivalent, and write $a \sim_{r} b$, if $a^{\frac{1}{n}} \sim_{c} b^{\frac{1}{n}}$ for all $n \in \mathbb{N}$. This is not the same as $a \sim_{c} b$ on $\mathfrak{S}_{A}$, even in the case $A=M_{2}$. This may be seen to be an equivalence relation on $\mathfrak{S}_{A}$ using the fact at the end of the first paragraph of this section.

Remark. (1) If $a, b \in \mathfrak{S}_{A}$, with $a \sim_{r} b$, consider the four-tuple or 'context' $(a A a, b A b, a A b, b A a)$. If $a^{\frac{1}{3 n}}=x y, b^{\frac{1}{3 n}}=y x$ for some $x, y \in \operatorname{Ball}(A)$, then letting $x^{\prime}=x y x, y^{\prime}=y x y$, we have $a^{\frac{1}{n}}=x^{\prime} y^{\prime}$ and $b^{\frac{1}{n}}=y^{\prime} x^{\prime}$, and $x^{\prime} \in \overline{a A b}$ and $y^{\prime} \in$ $\overline{b A a}$. Since these roots of $a$ and $b$ constitute cai's for $A_{a}$ and $A_{b}$ respectively, $(\overline{a A a}, \overline{b A b}, \overline{a A b}, \overline{b A a})$ is a Morita context in the sense of [11].

(2) Suppose that $a^{\frac{1}{n}} \sim_{c} b^{\frac{1}{n}}$ for some $n \in \mathbb{N}$, so $a^{\frac{1}{n}}=x y, y x=b^{\frac{1}{n}}$. Then $a^{\frac{1}{n}} x=x y x=x b^{\frac{1}{n}}$. Thus $a x=\left(a^{\frac{1}{n}}\right)^{n} x=x\left(b^{\frac{1}{n}}\right)^{n}=x b$, and similarly $a^{m} x=x b^{m}$ for all $m \in \mathbb{N}$. So $f(a) x=x f(b)$ for any function $f$ in the disk algebra, and even for more general functions. In particular, $p_{a} x=x p_{b}$. Similar assertions apply to $y$.

Parts of the following theorem use operations in the containing $C^{*}$-algebra $B$. However it will be seen that it does not matter which containing $C^{*}$-algebra we use, since other parts such as (iii) do not use $B$ at all. The careful reader will also notice apparent redundancy in some of the following statements and proofs; however this is necessary to obtain the first part of the last assertion of the theorem (about being equivalent with the same $x$ and $y$ ).

Theorem 2.2. Suppose that $a, b \in \mathfrak{S}_{A}$, and let $c=a^{\frac{1}{2}}$ and $d=b^{\frac{1}{2}}$. TFAE:

(i) There exist $w, y \in A$ with $a=w y, b=y w$ and $|y|=|c|$.

(ii) There exist $x, y \in A$ with $a=x y, b=y x$ and $|y|=|c|$, and $x=x p_{b}$.

(ii) $^{\prime}$ There exist $x, y \in A$ with $a=x y, b=y x$ and $|y|=|c|$, and $\left|x^{*}\right|=\left|c^{*}\right|$.

(ii)" There exist $x, y \in A$ with $a=x y, b=y x$ and

$$
|y|=|c|,\left|y^{*}\right|=\left|d^{*}\right|,|x|=|d|,\left|x^{*}\right|=\left|c^{*}\right| .
$$

(ii) ${ }^{\prime \prime \prime}$ There exist $x, y \in A$ with $a=x y, b=y x$ and $x=c R$ and $y=S c$ for some contractions $R, S$ in $A^{* *}$ (or if one prefers, in $B^{* *}$ ).

(iii) For all $n \in \mathbb{N}$, there exist $x_{n}, y_{n} \in \operatorname{Ball}(A)$ with $a^{\frac{1}{n}}=x_{n} y_{n}, b^{\frac{1}{n}}=y_{n} x_{n}$, and the sequence $\left(y_{n} a\right)$ has a norm convergent subsequence.

(iv) There exists a partial isometry $v \in B^{* *}$ with $p_{a}=v^{*} v, p_{b}=v v^{*}$, and $v a, a v^{*} \in A$ and $b=v a v^{*}$.

(iv)' There exists $v \in \Delta\left(A^{* *}\right)$ with $p_{a}=v^{*} v$, and $v a \in A$, and $b=v a v^{*}$.

These imply that $\|a\|=\|b\|$. The partial isometry $v$ in (iv) above may be chosen to be open and in $\Delta\left(A^{\perp \perp}\right)$. Also, the clauses (ii),(ii) $)^{\prime}(\mathrm{ii})^{\prime \prime}$, (ii) ${ }^{\prime \prime \prime}$ are equivalent to each other with the same $x$ and $y$, and these imply that $x \in \overline{a A b}, y \in \overline{b A a}$. 
Proof. Note that (iv) clearly implies that $\|a\|=\|b\|$.

(ii) $^{\prime \prime \prime} \Rightarrow$ (ii) $)^{\prime}$ Given (ii) $)^{\prime \prime \prime}, a=x y=c R S c$, we may assume that $S=S p_{a}$ and $p_{a} R=R$. So $c R S a=c a$ and so $c R S=c R S p_{a}=c$. Thus $R S=p_{a} R S=p_{a}$. It follows by e.g. 30] that $S$ is a partial isometry and $R=S^{*}$, so that $y^{*} y=c^{*} S^{*} S c=$ $c^{*} p_{a} c=c^{*} c$. Similarly, $x x^{*}=c c^{*}$.

(ii $^{\prime} \Rightarrow(\text { ii })^{\prime \prime \prime}$ Obvious.

(ii) $\Rightarrow$ (ii)" Assume that there exist $x, y \in A$ with $a=x y, b=y x$ and $|y|=|c|$. Then $y=r(y)|c|=v c$, where $v=r(y) r(c)^{*}$. Hence $y=y p_{a}$, so that $r(y)^{*} r(y) \leq p_{a}$. However $r(y)^{*} r(y) \geq p_{a}$ since $a=x y$, so $r(y)^{*} r(y)=p_{a}$. Similarly, $r(y) r(y)^{*} \geq p_{b}$. Note that $v^{*} v=r(c) p_{a} r(c)^{*}=p_{a}$. So $v$ is a tripotent. Also, $v v^{*}=r(y) p_{a} r(y)^{*}=$ $r(y) r(y)^{*} \geq p_{b}$, and we will see momentarily that this is an equality.

Note that $x y=x v c=c^{2}$ implies $x v a=c a$, and hence $x v=x v p_{a}=c p_{a}=c$. Since $y=v c$ we have $v a=v c^{2}=y c=y x v=b v$. Hence $y=v c=d v$. It is now clear that $p_{b} y=y$, so that $p_{b} \geq r(y) r(y)^{*}$, giving $p_{b}=r(y) r(y)^{*}=v v^{*}$. Also $y y^{*}=d v v^{*} d^{*}=d p_{b} d^{*}=d d^{*}$.

We now see that $x=c v^{*}$, assuming that $x=x p_{b}$ (since then $c v^{*}=x v v^{*}=x p_{b}=$ $x$ ). Thus $x x^{*}=c c^{*}$. By symmetry to the case for $y$, we must have $x^{*} x=c^{*} c$.

(ii) $\Rightarrow$ (iv) We use facts from the proof above, noting that $v a=v c^{2}=y c \in$ $A, a v^{*}=c^{2} v^{*}=c x \in A$, and $v a=v c^{2}=d^{2} v=b v$, so that $b=v a v^{*}$.

We now prove the assertions at the end of the theorem, starting from (ii), and using the notation and facts already established above. To see that $v$ is open, consider $R_{r(c)^{*}}$, right multiplication by $r(c)^{*}$, on the TRO $\langle y\rangle$ generated by $y$. This is a one-to-one ternary morphism, since $y r(c)^{*} r(c)=y p_{a}=y$. We show it also maps into $B$ : note $y r(c)^{*} c=y|c| \in B$, so $y r(c)^{*} a \in B$, hence $\operatorname{yr}(c)^{*} a^{\frac{1}{n}} \in \operatorname{yr}(c)^{*} \overline{a A} \subset B$. However $y r(c)^{*}=r(y)|c| r(c)^{*}=r(y) c^{*}$. Now $c^{*}\left(a^{\frac{1}{n}}\right)^{*} \rightarrow c^{*}$, so $c^{*} a^{\frac{1}{n}} \rightarrow c^{*}$ by 2.1 .6 in [10]. Hence $y r(c)^{*}=r(y) c^{*}=\lim _{n} r(y) c^{*} a^{\frac{1}{n}} \in B$. So $R_{r(c)^{*}}(\langle y\rangle) \subset B$. Hence $v$, the image under this ternary morphism of $r(y)$, is open, since $r(y)$ is open.

As we saw two paragraphs back, $v a \in A$ and $a v^{*} \in A$. By Lemma 1.2 we have $v a^{\frac{1}{n}} \in A$. In the weak* limit, $v=v p_{a} \in A^{* *}$. Similarly, $a^{\frac{1}{n}} v^{*} \in A$ and so $v^{*}=p_{a} v^{*} \in A^{* *}$. So $v \in \Delta\left(A^{* *}\right)$.

By Proposition [1.6, $x \in{ }_{p_{a}} A_{p_{b}}=\overline{a A b}$. Similarly, $y \in \overline{b A a}$.

(ii) $^{\prime} \Rightarrow$ (ii) As in the proof above, we have $v=r(y) r(c)^{*}$ is a tripotent with $y=v c, v^{*} v=p_{a}, v v^{*}=r(y) r(y)^{*}=p_{b}$. By symmetry, $w=r(c)^{*} r(x)$ is a tripotent with $x=c w, w w^{*}=p_{a}, w^{*} w=r(x)^{*} r(x)=p_{b}$. Now $a=x y=c w v c$, and by the idea in the proof of (ii) $)^{\prime \prime \prime} \Rightarrow(\text { ii })^{\prime}$ above, it follows that $w=v^{*}$, so that $x=c v^{*}=c v^{*} p_{b}=x p_{b}$.

(iv) $\Rightarrow$ (ii) ${ }^{\prime}$ Set $x=c v^{*}, y=v c$. These are in $A$ by Lemma 1.2 The rest is easy from facts in the proof of the last two implications.

(i) $\Rightarrow(\text { ii })^{\prime}$ Let $x=w y w, y^{\prime}=y w y$. Then $\left|y^{\prime}\right|^{2}=a^{*} y^{*} y a=\left|a^{\frac{3}{2}}\right|^{2}$. The proof that (ii) implies (ii)" and (iv) above, with $a, b$ replaced by $a^{3}, b^{3}$, yields that $x=a^{\frac{3}{2}} v, y^{\prime}=$ $v a^{\frac{3}{2}}$ for a partial isometry $v$ satisfying $v^{*} v=s(a)=s\left(a^{3}\right), v^{*} v=s(b)=s\left(b^{3}\right)$, and $v a^{3}=b^{3} v$, which implies that $v c=d v$. Since $c \in \overline{a A}$ and $a A=c c A \subset \overline{c A} \subset \overline{c^{3} A}$,

$$
v c \in v \overline{a A} \subset v \overline{c^{3} A} \subset \overline{v c^{3} A}=\overline{y^{\prime} A} \subset A .
$$

Similarly, $c v^{*} \in A$. Then $a=c v^{*} v c, b=d v v^{*} d=v c^{2} v^{*}$ as desired in (ii)' .

(ii) $\Rightarrow$ (i) Trivial.

(ii) $\Rightarrow$ (iii) Using the notation and proof above, if $y_{n}=v a^{\frac{1}{2 n}}, x_{n}=a^{\frac{1}{2 n}} v^{*}$, then these are in $A$ by considerations used above, and $x_{n} y_{n}=a^{\frac{1}{n}}$. As we said above, 
$v a=b v$, hence $v a^{n}=b^{n} v$, and so $v a^{\frac{1}{n}}=b^{\frac{1}{n}} v$ by considering the power series in Lemma 1.2. Hence $y_{n} x_{n}=b^{\frac{1}{2 n}} v v^{*} b^{\frac{1}{2 n}}=b^{\frac{1}{n}}$.

(iii) $\Rightarrow$ (iv) $)^{\prime}$ Note that $y_{n} a^{\frac{1}{n}}=y_{n} x_{n} y_{n}=b^{\frac{1}{n}} y_{n}$ so that $y_{n} a=b y_{n}$. Similarly, $a x_{n}=x_{n} b$. Replacing $y_{n}$ by $y_{n} x_{n} y_{n}$ and $x_{n}$ by $x_{n} y_{n} x_{n}$ as before, but still calling them $y_{n}$ and $x_{n}$, we have $a^{\frac{3}{n}}=x_{n} y_{n}$ where $x_{n}=p_{a} x_{n}, y_{n}=y_{n} p_{a}$, and we still have $y_{n} a=b y_{n}$ and $a x_{n}=x_{n} b$, and $\left(y_{n} a\right)$ still has a convergent subsequence. Suppose that $x_{n_{k}} \rightarrow S$ and $y_{n_{k}} \rightarrow T$ weak*, and $y_{n_{k}} a \rightarrow T a$ in norm. We have $S=p_{a} S$ and $T=T p_{a}$. Then $x_{n_{k}} y_{n_{k}} a \rightarrow S T a=p_{a} a=a$. As in the proof of (ii) $)^{\prime \prime \prime} \Rightarrow(\text { ii) })^{\prime}$, this yields $S T=S T p_{a}=p_{a}$, and $S^{*}=T$. Thus $T$ is a partial isometry $v \in \Delta\left(A^{* *}\right)$, with $v^{*} v=p_{a}$. Since $y_{n_{k}} a \rightarrow T a$ in norm, $v a \in A$. Since $y_{n} a=b y_{n}$ and $a x_{n}=x_{n} b$, it follows that $b y_{n_{k}} x_{n_{k}}=y_{n_{k}} a x_{n_{k}} \rightarrow v a v^{*}$, so that $b=v a v^{*}$. Hence $p_{b} \leq v v^{*}$, and in fact they are equal because $p_{b} v v^{*}=v v^{*}$ since $p_{b} y_{n}=y_{n}$. Thus we have (iv)'.

(iv) $^{\prime} \Rightarrow$ (iv) By Lemma 1.4, $b^{\frac{1}{n}}=\left(v a v^{*}\right)^{\frac{1}{n}}=v a^{\frac{1}{n}} v^{*}$. Then

$$
p_{b}=\lim _{n} b^{\frac{1}{n}}=\lim _{n} v a^{\frac{1}{n}} v^{*}=v p_{a} v^{*}=v v^{*},
$$

where these are weak* limits. If $v a \in A$ then $v B_{p}=v \overline{a B a} \subset B$, where $p=p_{a}$. Hence $B_{p} v^{*} \subset B$, so that $a v^{*} \in B \cap A^{* *}=A$.

Definition 2.3. Let $a, b \in \mathfrak{S}_{A}$. We say that $a$ is Pedersen equivalent to $b$ in $A$, and write $a \sim_{A} b$, if the equivalent conditions in the last theorem hold. We will see some other characterizations of this variant of Pedersen equivalence later in our paper. Sometimes we write $a \sim_{A} b$ via $x$ and $y$, if Theorem2.2 (ii)" (or equivalently (ii)) holds. Similarly if $n \in \mathbb{N}$ we write $a^{n} \sim_{A} b^{n}$ if (ii)" in Theorem 2.2 holds with $a$ and $b$ replaced by $a^{n}, b^{n}$. We will see that this happens iff $a \sim_{A} b$. If $a, b \in \mathfrak{c}_{A}$ with $\|a\|=\|b\|$, then we say that $a \sim_{A} b$ if some positive scalar multiple of $a$ is Pedersen equivalent in the sense above to the same multiple of $b$.

It should be admitted at the outset that our Pedersen equivalence (which generalizes the $C^{*}$-algebra variant-see Remark (2) below) happens more rarely in general operator algebras than it does in $C^{*}$-algebras. However this is similar, for example, to the fact that right ideals with left contractive approximate identities are rarer in general operator algebras than in $C^{*}$-algebras, but are still interesting (they are related to important topics, and have a compelling theory-see e.g. [9, 15]. While some algebras may have none, in other algebras they can be key for some purposes). Results such as Proposition 3.9, Theorem 4.9, and Theorem 5.1 show that our Pedersen equivalence happens quite naturally.

Remark. (1) Of course if $a, b \in \operatorname{Ball}\left(A_{+}\right)$in the case $A$ is a $C^{*}$-algebra, then $a \sim_{A} b$ iff there exists $x \in A$ with $a=x x^{*}, b=x^{*} x$ (the usual Pedersen equivalence). This is quite obvious; for example if $a \sim_{A} b$, with notation as in Theorem 2.2 (ii)", then $y^{*} y=b^{\frac{1}{2}} b^{\frac{1}{2}}=b$ and $y y^{*}=a^{\frac{1}{2}} a^{\frac{1}{2}}=a$.

If $A$ is a $C^{*}$-algebra then unitary equivalence of elements $a, b \in \mathfrak{S}_{A}$ implies $a \sim_{A} b$. Indeed if $b=u a u^{*}$ for a unitary $u$ in the multiplier algebra of $A$ (or simply a unitary in $A^{* *}$ such that $\left.a u^{*}, u a \in A\right)$, then $\operatorname{Ran}(b)=u \operatorname{Ran}(a)$. Thus $u p_{a}=p_{b} u p_{a}$. If we set $x=a^{\frac{1}{2}} u^{*}$ and $y=u a^{\frac{1}{2}}$ then $x y=a^{\frac{1}{2}} u^{*} u a^{\frac{1}{2}}=a$. Also, $y x=u a u^{*}=b$, and $x x^{*}=a^{\frac{1}{2}} u^{*} u\left(a^{\frac{1}{2}}\right)^{*}$. So $a \sim_{A} b$.

(2) From Theorem 2.2 (iii) we see that in a finite dimensional operator algebra $A, a \sim_{A} b$ iff $a \sim_{r} b$. Here $a, b \in \mathfrak{S}_{A}$. We do not know if this is true in general.

(3) The relation $\sim_{A}$ in the case that $A=M_{n}$ is exactly unitary equivalence of elements of $\mathfrak{S}_{M_{n}}$. The one direction of this follows from (1). For the other 
direction, if $a \sim_{A} b$ then we can get $b=w^{*} a w$ for a tripotent $w$ that the reader can easily check may be replaced by a unitary. Unitary equivalence and similarity are the same for positive matrices, but this is false for $\mathfrak{S}_{M_{n}}$ (even for $2 \times 2$ upper triangular matrices).

(4) We mention three other interesting ways to state Pedersen equivalence: (a) Theorem 2.2 (ii)" is equivalent with the same $x$ and $y$ to the same condition, but with $\left|x^{*}\right|=\left|c^{*}\right|$ replaced by $|x|=|d|$. Clearly this is implied by (ii)", and conversely it implies (ii), since $x=r(x)|x|=r(x)|d|=r(x) r(d)^{*} d$, which gives $x=x p_{b}$.

(b) $a \sim_{A} b$ iff there exist $x, y \in A$ with $a=x y, b=y x$, and for each $n \in \mathbb{N}$ there exist contractions $x_{n}, y_{n}$ with $x=c^{1-\frac{1}{n}} x_{n}, y=y_{n} c^{1-\frac{1}{n}}$. The $x_{n}, y_{n}$ can be chosen in $A$ if one wishes. To see that this is equivalent to (ii) ${ }^{\prime \prime \prime}$, in the one direction simply let $x_{n}=c^{\frac{1}{n}} R, y_{n}=S c^{\frac{1}{n}}$. For the other direction, if subnets $x_{n_{k}} \rightarrow R$ and $y_{n_{k}} \rightarrow S$ weak*, then (ii) ${ }^{\prime \prime \prime}$ holds. This is because by the continuity of the disk algebra functional calculus defining powers in [15, Section 2], it is easy to argue that $c^{1-\frac{1}{n}} \rightarrow c$ in norm.

(c) $a \sim_{A} b$ iff $a \sim_{r} b$ with some kind of 'compatibility' between the $x$ and $y$ elements for different roots. That is, for all $n \in \mathbb{N}$ one may write $a^{\frac{1}{n}}=x_{n} y_{n}, b^{\frac{1}{n}}=$ $y_{n} x_{n}$, where $x_{n}, y_{n}$ satisfy some conditions like $y_{k} x_{\ell}=y_{r} x_{s}$ whenever $\frac{1}{k}+\frac{1}{\ell}=\frac{1}{r}+\frac{1}{s}$; or like $y_{k} x_{\ell} y_{m}=y_{r} x_{s} y_{t}$ whenever $\frac{1}{k}+\frac{1}{\ell}+\frac{1}{m}=\frac{1}{r}+\frac{1}{s}+\frac{1}{t} \leq 1$. To see for example that $a \sim_{A} b$ is implied by the last condition, together with a couple of other reasonable conditions such as $x_{n}, y_{n}$ are contractions and $y_{n} \in \overline{b A a}$ : Note $y_{2 n} x_{2 n} y_{2}=b^{\frac{1}{2 n}} y_{2}$ converges to $y_{2}$, but it also equals $y_{n} x_{4} y_{4}=y_{n} a^{\frac{1}{4}}$. It follows that $\left(y_{n} a\right)$ converges, so Theorem 2.2 (iii) holds. Conversely, if Theorem 2.2 (ii) holds, let $x_{n}=a^{\frac{1}{2 n}} v^{*}, y_{n}=v a^{\frac{1}{2 n}}$. As in an argument in the proof of Theorem 2.2, these are in $A, x_{n} y_{n}=a^{\frac{1}{n}}, y_{n} x_{n}=b^{\frac{1}{n}}$, and

$$
y_{r} x_{s} y_{t}=v a^{\frac{1}{2 r}} a^{\frac{1}{2 s}} v^{*} v a^{\frac{1}{2 t}}=v a^{\frac{1}{2}\left(\frac{1}{r}+\frac{1}{s}+\frac{1}{t}\right)} .
$$

Thus $y_{k} x_{\ell} y_{m}=y_{r} x_{s} y_{t}$ if $\frac{1}{k}+\frac{1}{\ell}+\frac{1}{m}=\frac{1}{r}+\frac{1}{s}+\frac{1}{t} \leq 1$.

(5) Note that $p_{a}$ and $p_{b}$ are the left and right support projections of the element $x$ in Theorem 2.2 (ii) (and the variants of (ii)).

The choice of the square root, as opposed to a different root, in (i) and the variants of (ii) in Theorem 2.2 is not essential; one can instead take the $r$ th power and the $(1-r)$ th power, for any $r \in(0,1)$. For example:

Proposition 2.4. If $a, b \in \mathfrak{S}_{A}$ then $a \sim_{A} b$ iff there exist $x, y \in A$ with $a=x y, b=$ $y x$ and $|y|=\left|a^{r}\right|$ for some $r \in(0,1)$ and $x=x p_{b}$; or iff there exist $x, y \in A$ with $a=x y, b=y x$ and $|y|=\left|a^{r}\right|$ and $\left|x^{*}\right|=\left|\left(a^{1-r}\right)^{*}\right|$ for some $r \in(0,1)$.

Proof. Exactly as in the proof of Theorem 2.2 but replacing $c$ by $a^{r}$. For example, given (iv) in Theorem 2.2, let $y=v a^{r}, x=a^{1-r} v^{*}$, then it is easy to see, by the same arguments as in the proof of Theorem 2.2 that $x, y \in A$ with $a=x y, b=y x$, and $y^{*} y=\left(a^{r}\right)^{*} v^{*} v a^{r}=\left(a^{r}\right)^{*} p_{a} a^{r}=\left|a^{r}\right|^{2}$, so that $|y|=\left|a^{r}\right|$, and $x=x p_{b}$. Conversely, if $x, y \in A$ with $a=x y, b=y x$ and $|y|=\left|a^{r}\right|$, and $x=x p_{b}$, then all of the arguments in the first paragraph of the proof that (ii) $\Rightarrow$ (ii)" go through with $c$ replaced by $a^{r}$. In the second paragraph of that proof, one sees that $x v a^{r}=a^{1-r} a^{r}$, hence $x v a=a^{1-r} a$, so that $x v=a^{1-r}$. Also $v a=b v$, since $v a=v a^{r} a^{1-r}=y a^{1-r}=$ $y x v=b v$. One can modify the lines that follow in the proof we are mimicking, to get $y=v a^{r}=b^{r} v, p_{b}=v v^{*}$, and $y y^{*}=a^{r}\left(a^{r}\right)^{*}$. Thus $a^{1-r} v^{*}=x v v^{*}=x$, and so 
$x x^{*}=a^{1-r}\left(a^{1-r}\right)^{*}$. This all is more than enough to obtain (iv) in Theorem 2.2 indeed $v a=v a^{r} a^{1-r}=y a^{1-r} \in A, a v^{*}=a^{r} a^{1-r} v^{*}=a^{r} x \in A$. Since $v a=b v$, we have $b=v a v^{*}$.

Proposition 2.5. Suppose that $a, b \in \mathfrak{S}_{A}$ and $r \in(0,1) \cup \mathbb{N}$. Then $a \sim_{A} b$ iff $a^{r} \sim_{A} b^{r}$.

Proof. If $a \sim_{A} b$, then using notation from the proof of Theorem 2.2 above, let $y^{\prime}=v c^{r}, x^{\prime}=c^{r} v^{*}$. Note that $y^{\prime}=v c^{r} \in v \overline{a A a} \subset A$ since $y=v c \in A$. Similarly $x^{\prime} \in A$. Then $x^{\prime} y^{\prime}=c^{2 r}=a^{r}$, and $y^{\prime} x^{\prime}=v c^{2 r} v^{*}=v a^{r} v^{*}=b^{r}$. The latter follows by Lemma 1.4 and Lemma 1.3. So $a^{r} \sim_{A} b^{r}$.

The converse is very similar, suppose that $a^{r} \sim_{A} b^{r}$ via $a^{\frac{r}{2}} v^{*}, v a^{\frac{r}{2}}$, so that $b^{r}=v a^{r} v^{*}$. Let $x=a^{\frac{1}{2}} v^{*}, y=v a^{\frac{1}{2}}$. Then $x, y \in A$ and $x y=a$ as before, and

$$
y x=v a v^{*}=\left(\left(v a v^{*}\right)^{r}\right)^{\frac{1}{r}}=\left(v a^{r} v^{*}\right)^{\frac{1}{r}}=\left(b^{r}\right)^{\frac{1}{r}}=b,
$$

as can be easily argued following the idea in the last paragraph.

Corollary 2.6. $\sim_{A}$ is an equivalence relation on $\mathfrak{S}_{A}$.

Proof. We leave this as an exercise using e.g. Theorem 2.2 (iv).

Theorem 2.7. Suppose that $a \in \mathfrak{S}_{A}$, and let $c=a^{\frac{1}{2}}$. If there exists $x, y \in A$ with $a=x y$, and $|y|=|c|$ and $\left|x^{*}\right|=\left|c^{*}\right|$, then $b=y x$ is in $\mathfrak{S}_{A}$, and $a \sim_{A} b$.

Proof. Using the notation and proof of (ii) ${ }^{\prime} \Rightarrow$ (ii) in Theorem 2.2 above, we have $y=v c$, and hence $v v^{*} b=b$, and $x=c w$ and $b w^{*} w=b$. Following that proof, we see that $w=v^{*}$ again, so that $b=v v^{*} b v v^{*}$. So

$$
\|1-2 b\|=\left\|v v^{*}-2 v v^{*} b v v^{*}\right\| \leq\left\|1-2 v^{*} y x v\right\|=\left\|1-2 c^{2}\right\|=\|1-2 a\| .
$$

Similarly, $\|1-b\| \leq\|1-a\|$. So $b=y x$ is in $\mathfrak{S}_{A}$, and $a \sim_{A} b$.

\section{Open tripotents for operator Algebras And Peligrad-Zsidó EQUIVALENCE}

The partial isometry or tripotent $v$ that occurs in Theorem 2.2 is central to our study of the above variant of Pedersen equivalence. It also happens to be what we will call a $*$-open tripotent. In the present section we define this notion and develop the associated theory. Later we will apply this theory back to Pedersen equivalence, and to coarser notions of equivalence and subequivalence.

In the introduction we discussed the notion from [12] of an open tripotent in $B^{* *}$, and gave several equivalent definitions of these objects. We will also use below the following characterization, which is proved below in the Appendix (Proposition 6.1): A partial isometry $v$ in $B^{* *}$ is an open tripotent in the sense of [12] iff $p=v^{*} v$ and $q=v v^{*}$ are open projections in the sense of Akemann, and $v B_{p} \subset B$ and $v^{*} B_{q} \subset B$. As we said in the introduction, first, $p$ and $q$ are said to be PeligradZsidó equivalent in this case, and second, the conditions involving $q$ in the last line are automatic and are stated for the sake of symmetry.

We define a *-open tripotent (or *-open partial isometry) in $A^{* *}$, for an operator algebra $A$, to be an open tripotent (in the sense of [12]) in $B^{* *}$, which lies in $\Delta\left(A^{\perp \perp}\right)$. Here $B$ is a $C^{*}$-algebra generated by $A$.

Lemma 3.1. If $v$ is an open tripotent in $B^{* *}$ then so is $v^{*}$. Hence if $v$ is a*-open tripotent in $A^{* *}$ then so is $v^{*}$. 
Proof. We leave this as an exercise.

Being a $*$-open tripotent makes sense independently of the particular $C^{*}$-algebra $B$ generated by $A$, since one may define '*-openness' in $A^{* *}$ purely in terms of $A$ : $u$ is a $*$-open tripotent in $A^{* *}$ iff $u$ is a tripotent in the $C^{*}$-algebra $\Delta\left(A^{* *}\right)$, and the projection

$$
\hat{u}=\frac{1}{2}\left[\begin{array}{cc}
u u^{*} & u \\
u^{*} & u^{*} u
\end{array}\right]
$$

in $M_{2}\left(\Delta\left(A^{* *}\right)\right)$, is an open projection in $M_{2}(A)^{* *}$ in the sense of [9] (that is, there exists a net $a_{t} \in M_{2}(A)$ such that $a_{t}=\hat{u} a_{t}=a_{t} \hat{u} \rightarrow \hat{u}$ weak* in $\left.M_{2}(A)^{* *}\right)$. This is also equivalent to $\hat{v}$ being open in $M_{2}(A)^{* *}$ in the sense of [9], where $v=u^{*}$.

Remark. (1) Open projections in $A^{* *}$ in the sense of 9 , are clearly *-open tripotents in $A^{* *}$. This follows from the definition above since open projections in $B^{* *}$ are open tripotents in the sense of $[12$.

(2) It is easy to see how to define a $*$-open tripotent in the second dual of a strong Morita equivalence bimodule $X$ in the sense of [11, and a matching Peligrad-Zsidó equivalence for open projections in the second duals of the algebras acting on the left and right of $X$. Most of the results below about $*$-open tripotents and PeligradZsidó equivalence will be almost identical in this more general setting, with almost identical proofs, and we leave this to the reader. This will then simultaneously generalize much of the theory in the present paper, and the theory of open tripotents in 12. We have chosen not to present this here, since it is often better to present a more concrete theory than to present the most general abstract formulation. We do remark however that many results in this more general theory are derivable from the case we consider by taking $A=\mathcal{L}(X)$, the linking algebra of $X$.

We say that two projections $p, q \in A^{* *}$ are Peligrad-Zsidó equivalent in $A^{* *}$, and write $p \sim_{P Z, A} q$ if there exists an open tripotent (in the sense of 12] $v \in B^{* *}$, which lies in $\Delta\left(A^{\perp \perp}\right)$ (hence is $*$-open), implementing a Peligrad-Zsidó equivalence of $p$ and $q$ in $B^{* *}$. That is, $v^{*} v=p, v v^{*}=q$ (since $v$ is open this forces $p, q$ to be open projections [12]).

Remark. If $p \sim_{B, P Z} q$ it does not follow that $p \sim_{P Z, A} q$ necessarily. A simple counterexample is the diagonal matrix units in the case $A$ is the upper triangular 2 by 2 matrices.

To connect our definition to Pelegrad and Zsidó's original equivalence, as we did in the selfadjoint case (see Proposition 6.1), we have:

Lemma 3.2. Two projections $p, q \in A^{* *}$ are Peligrad-Zsidó equivalent in $A^{* *}$ iff $p, q$ are open projections and there exists $v \in B^{* *}$ such that $v^{*} v=p, v v^{*}=q$, and $v A_{p} \subset A$ and $v^{*} A_{q} \subset A$. This is also equivalent to: $p$ is open and there exists $v \in \Delta\left(A^{* *}\right)$ such that $v^{*} v=p, v v^{*}=q$, and $v A_{p} \subset A$.

Proof. If $p \sim_{P Z, A} q$ via $v$ then as we said above, $p, q$ are open. Also by Proposition 6.1 from the Appendix, $v A_{p} \subset v B_{p} \subset B \cap A^{\perp \perp}=A$, and similarly $v^{*} A_{q} \subset A$.

Conversely, suppose that $p, q$ are open and that $v A_{p} \subset A$ and $v^{*} A_{q} \subset A$. Then $v e_{t} \in A$ if $\left(e_{t}\right)$ is a cai for $A_{p}$. Taking a weak* limit, $v p=v \in A^{\perp \perp}$. Similarly, $v^{*} \in A^{\perp \perp}$, so $v \in \Delta\left(A^{\perp \perp}\right)$. Now $\left(e_{t}\right)$ is also a cai for $B_{p}$ by [9, Theorem 2.9], so it follows that for any $c \in B_{p}$ we have $v c=\lim _{t} v e_{t} c \in A C \subset B$. That is, $v B_{p} \subset B$. Similarly, $v^{*} B_{q} \subset B$, so by Proposition 6.1 from the Appendix, $v$ implements a 
Peligrad-Zsidó equivalence of $p_{a}$ and $p_{b}$ in $B^{* *}$. If $p$ is open and $v A_{p} \subset A$ then the argument above gives $v B_{p} \subset B$. So by [34, Lemma 1.3], $q$ is open and $v^{*} B_{q} \subset B$. Thus if $v^{*} \in A^{\perp \perp}$ then $v^{*} A_{q} \subset B \cap A^{\perp \perp}=A$.

Remark. Under the equivalent conditions of the Lemma, we also have $A_{q} v \subset$ $B_{q} v=\left(v^{*} B_{q}\right)^{*} \subset B$, so $A_{q} v \subset B \cap A^{\perp \perp}=A$. Similarly, $A_{p} v^{*} \subset A$.

Theorem 3.3. Let $u$ be a tripotent in $A^{\perp \perp}$, and let

$$
A_{u}=\left\{a \in A: a=u u^{*} a u^{*} u\right\} .
$$

Suppose that $u$ is in the weak closure of $A_{u}$, and that $A_{u} u^{*} \subset A$. Then $u$ is an open tripotent in $B^{* *}$. If, further, $u^{*} \in A^{\perp \perp}$ then $u$ is a $*$-open tripotent in $A^{* *}$, $u^{*} A_{u} \subset A$, and the weak* closure of $A_{u}$ equals

$$
\left\{\eta \in A^{* *}: \eta=u u^{*} \eta u^{*} u\right\}=u u^{*} A^{* *} u^{*} u .
$$

Conversely, if $u$ is a $*$-open tripotent in $A^{* *}$ then $u$ is in the weak ${ }^{*}$ closure of $A_{u}$, and $A_{u} u^{*} \subset A$.

Proof. We have $u A_{u}^{*} A_{u} \subset B$. As explained in the introduction, $B_{2}^{* *}(u)$ is a $C^{*}$ algebra with product $x u^{*} y$ and involution $u x^{*} u$. In this product and involution the $C^{*}$-algebra generated by $u A_{u}^{*} A_{u}$ is contained in $B$. The increasing cai for this $C^{*}$-algebra converges to a projection in the $C^{*}$-algebra $B_{2}^{* *}(u)$, hence to a tripotent $w \in B^{* *}$. Since $w u^{*} w=w$, we have $w \leq u$ in the ordering of tripotents. It is an exercise to check that $w$ satisfies the definition from [12] of an open tripotent in $B^{* *}$. If $x, y \in A_{u}$ we have $u x^{*} y=w u^{*} u x^{*} y=w w^{*} u x^{*} y=w x^{*} y$. Replacing $x$ with $x_{t} \in A_{u}$ such that $x_{t} \rightarrow u$ weak* $^{*}$, we have $u u^{*} y=w u^{*} y=w w^{*} y$. Replacing $y$ with $x_{t}$ in the limit we have $u=u u^{*} u=w w^{*} u=w$. So $u$ is open in $B^{* *}$.

If further $u^{*} \in A^{\perp \perp}$ then $u^{*} A_{u} \subset u^{*} B_{u} \subset B \cap A^{* *}=A$. Clearly $u$ is *-open in $A^{* *}$. The weak* closure of $A_{u}$ is contained in $u u^{*} A^{* *} u^{*} u$ clearly. Conversely, if $p=u^{*} u$, which is open in $A^{* *}$ by [12], and $\eta \in u u^{*} A^{* *} u^{*} u$, then $u^{*} \eta \in p A^{* *} p$, so there is a net $\left(a_{t}\right)$ in ${ }_{p} A_{p}$ converging weak* to $u^{*} \eta$. Then $u a_{t} \rightarrow \eta$ weak $^{*}$, and $u a_{t} \in A$, indeed $u a_{t} \in A_{u}$. So the weak* closure of $A_{u}$ is $u u^{*} A^{* *} u^{*} u$.

For the converse, if $u$ is a $*$-open tripotent in $A^{* *}$ then by the fact above the theorem there exists a net $x_{t} \in A_{u}$ with $x_{t} \rightarrow u$ weak*. If $x \in A_{u}$ then $x \in B_{u}$. In the notation of [12, $B_{u} u^{*}=B(u) u^{*} \subset B$ by facts in [12. Then $x u^{*} \in B_{u} u^{*} \cap A^{* *} \subset$ $B \cap A^{* *}=A$.

In the following, $\mathfrak{S}_{A_{u}}$ is computed with respect to operator algebra $A_{u}$ with the Peirce product. That is, $\mathfrak{S}_{A_{u}}=\left\{a \in A_{u}:\|u-K a\| \leq 1\right\}$, where $K=1$ or 2 .

Lemma 3.4. If $u$ is a $*$-open tripotent in $A^{* *}$ then $A_{u}$ is a subalgebra of the $C^{*}$ algebra $B(u)$ with its 'Peirce product', and it has a cai with respect to this product. If $u, w$ are $*$-open tripotents in $A^{* *}$, then TFAE:

(i) $u \leq w$ in the ordering of tripotents,

(ii) $A_{u}$ is a subalgebra (indeed is a HSA) of $A_{w}$ in the product above,

(iii) $\mathfrak{S}_{A_{u}} \subset \mathfrak{S}_{A_{w}}$ as sets,

(iv) $\hat{u} \leq \hat{w}$.

Indeed a tripotent in $A^{* *}$ which is dominated by $w$ in the ordering of tripotents, is $*$-open in $A^{* *}$ iff it is an open projection in the operator algebra $A_{w}^{* *}$ (with its Peirce product). Moreover, $u=w$ iff $A_{u}=A_{w}$ as sets and as algebras (with the product above). 
Proof. That $A_{u}$ is a subalgebra of $B(u)$ follows from the result $A_{u} u^{*} \subset A$ above. Since the second dual of $A_{u}$ is its weak* closure $u u^{*} A^{* *} u^{*} u$, which contains $u$ which is an identity in the 'Peirce product', it follows from e.g. [10, Proposition 2.5.8] that $A_{u}$ has a cai.

If $u \leq w$ then it is clear that $A_{u} \subset A_{w}$ as sets.

(i) $\Rightarrow$ (ii) If $x, y \in A_{u}$ then $x w^{*} y=x u^{*} u w^{*} y=x u^{*} y$, so $A_{u}$ is a subalgebra of $A_{w}$. We leave it as an exercise that it is a HSA.

(ii) $\Rightarrow$ (i) If $A_{u}$ is a subalgebra of $A_{w}$ then $\left(A_{u}\right)^{* *}$ is a subalgebra of $\left(A_{w}\right)^{* *}$. Thus $u w^{*} u=u$, so $u \leq w$.

(i) $\Leftrightarrow$ (iv) is in [12, Proposition 2.7].

(ii) $\Rightarrow$ (iii) If (ii) holds then by (i) $u$ may be viewed as a projection in $\left(A_{w}\right)^{* *}$ dominated by the identity $w$. Let $x \in A_{u} \subseteq A_{w}$ with $\|u-2 x\| \leq 1$. Then $\|w-2 x\|=\|w-u+u-2 x\| \leq \max \{1,\|u-2 x\|\} \leq 1$.

(iii) $\Rightarrow$ (i) Taking second duals, and using [15, Lemma 8.1] we have $\mathfrak{S}_{A_{u}^{* *}} \subset \mathfrak{S}_{A_{w}^{* *}}$. By the fact mentioned in the introduction that $\mathfrak{c}_{A}$ densely spans $A$, we have that $A_{u}^{* *} \subset A_{w}^{* *}$. In particular, $\|w-2 u\| \leq 1$ since $\|u-2 u\| \leq 1$. We wish to show that $u w^{*} u=u$, or equivalently that $u$ is a projection in the $C^{*}$-algebra $C=B_{2}^{* *}(w)$. Working inside the latter $C^{*}$-algebra we have $w=1$. Thus it suffices to prove that any partial isometry $V$ in a unital $C^{*}$-algebra $C$, with $\|1-2 V\| \leq 1$, is a projection. Since $V \in \mathfrak{S}_{C}$, its left support and right supports agree, so $V^{*} V=V V^{*}$ equals a projection $p=s(V)$. If $D=p C p$ then $V$ is a unitary in $D$ with $\|p-2 V\| \leq 1$. By spectral theory it follows that $V=p$. This completes the proof of (i).

Given an open projection $p \in\left(A_{w}\right)^{\perp \perp}$, we recall that $A_{w}$ is a subalgebra of $B(w) \subset B_{2}^{* *}(w)$. So by [9, Theorem 2.4], $p$ is open in $B_{2}^{* *}(w)$. By [12, Corollary 2.11], $p$ is an open tripotent $u$ in $B^{* *}$. We also have that $u^{*}=p^{*}=w^{*} p w^{*} \in A^{* *}$. So $u$ is a $*$-open tripotent in $A^{* *}$.

Conversely, any $*$-open tripotent $u \in A^{* *}$ is open as a tripotent in $B^{* *}$. If $u \leq w$, then $u \in\left(A_{w}\right)^{\perp \perp} \subset B_{2}^{* *}(w)$. By [12, Corollary 2.11], $u$ is open as a projection in $B_{2}^{* *}(w)$. Since $A_{w}$ is a subalgebra of $B(w)$ and $u \in\left(A_{w}\right)^{\perp \perp}, u$ is open as a projection in $\left(A_{w}\right)^{\perp \perp}$ by [9, Theorem 2.4].

Remark. If $A_{u}=A_{w}$ as subsets of $A$, it need not follow that $u=w$. This may be seen by considering for example any two distinct unitaries in $K(H)^{* *}=B(H)$. Indeed $A_{u}=A_{w}$ iff $u^{*} u=w^{*} w$ and $u u^{*}=w w^{*}$. To see the harder direction of this, if $A_{u}=A_{w}$ then in the second duals we see that $u \in B_{2}^{* *}(w)$, so that $u^{*} u \leq w^{*} w$ and $u u^{*} \leq w w^{*}$. Similarly, $w^{*} w \leq u^{*} u$ and $w w^{*} \leq u u^{*}$.

From Sections 2 and 3 of [12] one can write down a host of properties and behaviors of open tripotents in $A^{* *}$. For example:

Lemma 3.5. An increasing net of *-open tripotents in $A^{* *}$ has a least upper bound tripotent in $A^{* *}$, namely its weak ${ }^{*}$ limit, and this limit tripotent is $*$-open in $A^{* *}$.

Proof. Clear from [12, Proposition 2.12] and the characterization of $*$-open tripotents in $A^{* *}$ as open tripotents in $B^{* *}$ which are also in the von Neumann algebra $\Delta\left(A^{* *}\right)$.

Lemma 3.6. If $u$ and $v$ are $*$-open tripotents in $A^{* *}$, which are both dominated by some tripotent in $B^{* *}$, then there exists a smallest tripotent $u \vee v$ in $B^{* *}$ which dominates both $u$ and $v$, and this tripotent is a *-open tripotent in $A^{* *}$. 
Proof. Of course $u$ and $v$ are open tripotents in $B^{* *}$. That there exists a smallest tripotent $u \vee v$ in $B^{* *}$ which dominates both $u$ and $v$ is well known (see e.g. [12, Lemma 3.7]). By [12, Lemma 3.7], $\widehat{u \vee v}=\hat{u} \vee \hat{v}$. However $\hat{u}$ and $\hat{v}$ are open projections in $M_{2}\left(\Delta\left(A^{* *}\right)\right)$, so we deduce that $\widehat{u \vee v}$ is an open projection and is in $M_{2}\left(\Delta\left(A^{* *}\right)\right)$, so that $u \vee v$ is a $*$-open tripotent in $A^{* *}$.

Lemma 3.7. A family $\left\{u_{i}\right\}$ of $*$-open tripotents in $A^{* *}$, which are all dominated by a tripotent in $B^{* *}$, has a least upper bound amongst the tripotents in $B^{* *}$, and this is a *-open tripotent in $A^{* *}$.

Proof. Of course the $u_{i}$ are open tripotents in $B^{* *}$, so by [12, Proposition 3.8] the family has a least upper bound $v$ amongst the tripotents in $B^{* *}$, and this is an open tripotent in $B^{* *}$. The supremum of any finite subfamily of $\left\{u_{i}\right\}$ is a $*$-open tripotent by Lemma 3.6 and such supremums form an increasing net with least upper bound $v$. so $v$ is a $*$-open tripotent in $A^{* *}$ by Lemma 3.5 .

We now rephrase Pedersen equivalence in terms of $*$-open tripotents. We can rephrase a special case of Lemma 3.2 as follows. Let $v \in B^{* *}$ with $v^{*} v=p_{a}$ for some $a \in \mathfrak{S}_{A}$ with $v a \in A$. Then $v B_{p_{a}}=v \overline{a B a} \subset B$, and so $v$ is an open tripotent in $B^{* *}$, and $v=\lim _{n} v a^{\frac{1}{n}} \in A^{* *}$. In this case, $v$ is a $*$-open tripotent in $A^{* *}$ iff $v^{*} \in A^{* *}$, and iff $a v^{*} \in A$.

Proposition 3.8. If $a, b \in \mathfrak{S}_{A}$ then $a \sim_{A} b$ iff there exists $a$ *-open tripotent $v$ with $v^{*} v=p_{a}, v v^{*}=p_{b}$ and $b=v a v^{*}$. Also, in this case the $x$ and $y$ in Theorem 2.2 (ii) are in bijective correspondence with such $*$-open tripotents $v$ satisfying all the conditions in the last line. The bijection here takes $v$ to $y=v c\left(\right.$ and $\left.x=c v^{*}\right)$, and its inverse takes $y$ to $r(y) r(c)^{*}$. Here $c=a^{\frac{1}{2}}$.

Proof. The first assertion is clear from Theorem 2.2 (iv) and Lemma 3.2 (and the fact that $v a \in A$ implies that $v \overline{a A a} \subset A$ ). To finish we show that the two maps described are inverses of each other. If $y$ is as in Theorem 2.2 (ii) we have $r(y) r(c)^{*} c=r(y)|c|=r(y)|y|=y$. Conversely, if $v$ is a $*$-open tripotent satisfying all the conditions in the first line of the theorem statement, then $v c=v r(c)|c|=v r(c)|v c|$. Now $v r(c)$ is a tripotent, with left support $v s(c) v^{*}=v v^{*}$ and right support $r(c)^{*} v^{*} v r(c)=p_{a}$. By the 'uniqueness of the polar decomposition' we deduce that $v r(c)=r(v c)$. Thus $r(v c) r(c)^{*}=v r(c)^{*} r(c)=v p_{a}=v$. This completes the proof.

Proposition 3.9. Let $v$ be $a *$-open tripotent in $A^{* *}$, and suppose that $p=v^{*} v$ is the support projection for some $a \in \mathfrak{S}_{A}$. Then $q=v v^{*}$ is the support projection for some $b \in \mathfrak{S}_{A}$, and $a \sim_{A} b$.

Proof. Let $b=v a v^{*}$. As in [15, $\overline{a A a}=A_{p}$ since $p=s(a)$. Note that $b=$ $v a^{\frac{1}{2}} a^{\frac{1}{2}} v^{*} \in v A_{p} A_{p} v^{*} \subset A_{q}$, using the Remark after Lemma 3.2. Also, $b \in \mathfrak{S}_{A}$ and $\left(v a v^{*}\right)^{\frac{1}{n}}=v a^{\frac{1}{n}} v^{*}$ by Lemma 1.4. As at the end of the proof of Theorem 2.2. $p_{b}=v v^{*}=q$. That $a \sim_{A} b$ now follows from Proposition 3.8.

Remark. A similar result: suppose that $a \in \mathfrak{S}_{A}$, and that for all $n \in \mathbb{N}$, there exist $x_{n}, y_{n} \in \operatorname{Ball}(A)$ with $a^{\frac{1}{n}}=x_{n} y_{n}$, and the sequence $\left(y_{n} a\right)$ has a norm convergent subsequence with limit $v a$ say, where $v$ is a weak ${ }^{*}$ cluster point of $\left(y_{n}\right)$. Then $v$ is a $*$-open tripotent, $v \in \Delta\left(A^{* *}\right)$, and $b=v a v^{*} \in \mathfrak{S}_{A}$ with $a \sim_{A} b$. To prove this, suppose that $x_{n_{k}} \rightarrow S$ and $y_{n_{k}} \rightarrow v$ weak*, and $y_{n_{k}} a \rightarrow v a$ in norm. As usual 
we may assume that $y_{n}=y_{n} p_{a}, x_{n}=p_{a} x_{n}$, and $S=p_{a} S$ and $v=v p_{a}$. Then $x_{n_{k}} y_{n_{k}} a \rightarrow S v a=p_{a} a=a$, so that $S v=S v p_{a}=p_{a}$. This forces $S^{*}=v$, and $v$ is a tripotent in $\Delta\left(A^{* *}\right)$. Since $y_{n_{k}} a \rightarrow v a$ in norm, $v a \in A$. As in Lemma 3.2 above, $v B_{p} \subset B$, where $p=p_{a}$. By [34, Lemma 1.3], if $q=v v^{*}$ then $q$ is open and $v^{*} B_{q} \subset B$. So $v$ is open, and $a^{\frac{1}{2}} v^{*} \in B_{p} v^{*} \cap A^{\perp \perp}=A$, and similarly $v a^{\frac{1}{2}} \in A$. So by Proposition 3.9 we have $b=v a v^{*} \in \mathfrak{S}_{A}$ and $a \sim_{A} b$.

\section{Hereditary Bimodules}

Hereditary bimodules, defined below, as well as being interesting in their own right, will be important for us for two main reasons. First, they are useful in understanding Pedersen and Blackadar equivalence: if one examines the algebraic structure of $\overline{a A b}$ when $a$ and $b$ are equivalent in these ways, then one is led naturally to hereditary bimodules. (Indeed we give several characterizations of these equivalences in terms of hereditary bimodules in this section and the next.) Secondly, hereditary bimodules will turn out to be much the same thing as *-open tripotents $u$, via the correspondence $u \mapsto A_{u}$, with $A_{u}$ defined as in the previous section. One important definition of open tripotents from [12] is as the 'support tripotents' of what was called an inner $C^{*}$-ideal. Hereditary bimodules are the analogue of inner $C^{*}$-ideals in our setting, and we shall see in Proposition 4.1 below that $*$-open tripotents are the same as 'support tripotents' of hereditary bimodules.

We define a hereditary bimodule to be the 1-2 corner of a HSA in $M_{2}(A)$, where that HSA is isomorphic to $M_{2}(C)$ for an approximately unital operator algebra $C$, via a 'corner preserving' completely isometric homomorphism $\rho: M_{2}(C) \rightarrow M_{2}(A)$. Thus we have subalgebras $D, E$ of $A$, and subspaces $X, Y$ of $A$, such that the subalgebra $\mathcal{L}$ of $M_{2}(A)$ with rows $C, X$ and $Y, D$, is a HSA in $M_{2}(A)$, and there is a completely isometric isomorphism $\rho$ from $M_{2}(C)$ onto $\mathcal{L}$ taking each of the corners $D, E, X, Y$ of $\mathcal{L}$ onto the copy of $C$ in the matching corner of $M_{2}(C)$. In this case we say that $X$ is a hereditary bimodule, or a hereditary D-E-bimodule, and we call $\mathcal{L}$ the linking algebra of the hereditary bimodule. It then follows easily that $(D, E, X, Y)$ is a strong Morita equivalence context in the sense of [11, that $Y$ is the 'dual module' $\tilde{X}$ considered in that theory, $X=\tilde{Y}$, and that $\mathcal{L}$ is the linking algebra in the sense of [1] for this strong Morita equivalence. Conversely, given a strong Morita equivalence context $(D, E, X, Y)$ whose linking algebra $\mathcal{L}$ is a HSA in $M_{2}(A)$, then saying that $\mathcal{L}$ is isomorphic to $M_{2}(C)$ in the way described above, is, by [8, Corollary 10.4], the same as saying that the 1-2 corner $X$ is linearly completely isometric to an approximately unital operator algebra. That is, given a strong Morita equivalence context $(D, E, X, Y)$ whose linking algebra $\mathcal{L}$ is a HSA in $M_{2}(A)$, then $X$ is a hereditary bimodule iff $X$ is linearly completely isometric to an approximately unital operator algebra.

We point out that $D=X Y$ and $E=Y X$ above. These are HSA's in $A$. But also $X=D A E$ and $Y=E A D$, and these are inner ideals in $A$ (that is, $X A X \subset X$ and similarly for $Y$ ). So $X$ and $Y$ are retrievable from $D$ and $E$. To see this, note that clearly $X=D X E \subset D A E$, and conversely $D A E=X Y A Y X \subset X$ since $X$ is an inner ideal. So $X=D A E$, and similarly $Y=E A D$.

In the notation above, note that a hereditary bimodule $X$ is an approximately unital operator algebra in the product transferred from $C$ via $\rho$. The weak* limit in $A^{* *}$ of the cai for $X$ will be called a support tripotent of $X$ (in the proof of the next result it will be seen to be a tripotent). This support tripotent is not unique, as we 
discussed for example in the remark before Lemma 3.5. but can be easily be made so similarly to the case in [12] by keeping track of one further peice of structure on a hereditary bimodule, such as its 'product' (or one of the other items in Lemma 3.4).

We define a principal hereditary $A_{a}-A_{b}$-bimodule, or principal hereditary bimodule for short, to be a hereditary $A_{a}-A_{b}$-bimodule $X$ for some $a, b \in \mathfrak{S}_{A}$. We have $X=$ $\overline{a A b}$ by Proposition 1.6. The linking algebra $\mathcal{L}$ of the bimodule is the subalgebra of $M_{2}(A)$ with rows $\overline{a A a}, \overline{a A b}$ and $\overline{b A a}, \overline{b A b}$. Note that this $\mathcal{L}$ is certainly a HSA in $M_{2}(A)$.

Remark. For any $a, b \in \mathfrak{S}_{A}$, the subalgebra of $M_{2}(A)$ with rows $\overline{a A a}, \overline{a A b}$ and $\overline{b A a}, \overline{b A b}$, is a HSA in $M_{2}(A)$ clearly. However it will not in general correspond to a strong Morita equivalence. Indeed, if $X=\overline{a A b}$, there may be no HSA in $M_{2}(A)$ with $X$ as its 1-2 corner which corresponds to a strong Morita equivalence. Obvious counterexamples exist in the case $A$ is the upper triangular 2 by 2 matrices.

Proposition 4.1. If $u$ is a $*$-open tripotent in $A^{* *}$, then $A_{u}$ is a hereditary bimodule. Conversely, any hereditary bimodule equals $A_{u}$ for $a *$-open tripotent $u$; and $u$ is a support tripotent of this hereditary bimodule.

Proof. If $u$ is a *-open tripotent in $A^{* *}$ then by Lemma 3.1 so is $v=u^{*}$. Let $p=v^{*} v, q=v v^{*}$. The subalgebra $\mathcal{L}$ of $M_{2}(A)$ with rows ${ }_{p} A_{p},{ }_{p} A_{q}$ and ${ }_{q} A_{p},{ }_{q} A_{q}$, is a HSA of $M_{2}(A)$. Note ${ }_{p} A_{q}=A_{u}$. Since $A_{u}$ is an approximately unital operator algebra in the 'Peirce product', it is a hereditary bimodule by the definition of the latter. Explicitly, if $S=\operatorname{diag}(1, v)$, then $\ell \mapsto S^{*} \ell S$ is a completely contractive homomorphism from $\mathcal{L}$ to $M_{2}\left({ }_{p} A_{p}\right)$, whose completely contractive inverse is $k \mapsto$ $S k S^{*}$.

For the converse, we use the notation in our definition and discussion of 'hereditary bimodule' at the start of Section 4 . Let $e$ be the identity of $C^{* *}$. Note that $\rho^{* *}: M_{2}\left(C^{* *}\right) \rightarrow M_{2}\left(A^{* *}\right)$ is a completely isometric corner preserving homorphism too, and it restricts to an injective $*$-homomorphism from $M_{2}(\mathbb{C} e)$ into $\Delta\left(\mathcal{L}^{* *}\right)$. Note that the identity of $\mathcal{L}^{* *}$ is $p+q$ where $p$ is the support projection of $D$ and $q$ is the support projection of $E$. Also $p$ and $q$ correspond via $\rho^{* *}$ to $e \oplus 0$ and $0 \oplus e$ in $M_{2}\left(C^{* *}\right)$. Let $u$ be the image in $A^{* *}$ of $e$ under the 1-2 corner of $\rho^{* *}$. Then $v=u^{*}$ is the image in $A^{* *}$ of $e$ under the 2-1 corner of $\rho^{* *}$. Also, $u$ is a tripotent in $X^{\perp \perp}$, and $v v^{*}=q, v^{*} v=p$ (since the matching facts are true in $M_{2}(\mathbb{C} e)$ ). Also, $u A_{p} \subset A$, since the matching products are in $C$, and $\rho(C) \subset M_{2}(A)$. So $u$ is a *-open tripotent in $A^{* *}$ by Lemma 3.2 (one could also use Theorem 3.3 to see this). Since a cai for $C$ converges weak* to $e$, it is clear that $u$ is a support tripotent in the sense above.

The following is an intrinsic characterization of hereditary bimodules:

Theorem 4.2. An inner ideal $X$ in $A$ is a hereditary bimodule, or equivalently is of the form $A_{u}$ for a $*$-open tripotent $u$, if and only if there are nets of contractions $c_{n} \in X$ and $d_{n} \in A$, such that

(1) $c_{n} d_{n} x \rightarrow x$ and $x d_{n} c_{n} \rightarrow x$ in norm for all $x \in X$,

(2) $\left(d_{n} c_{m}\right)$ and $\left(c_{m} d_{n}\right)$ are norm convergent with $n$ for fixed $m$, and are norm convergent with $m$ for fixed $n$.

If these hold then a subnet of the $c_{n}$ converge weak ${ }^{*}$ to a support tripotent $u$ for $X$, so that $X=A_{u}$. 
Proof. $(\Rightarrow)$ Suppose that $u$ is a $*$-open tripotent, so that $A_{u}$ is a hereditary bimodule, isomorphic to an approximately unital operator algebra $C$. Then since these conditions clearly hold in $C$, by applying the isomorphism $\rho$ in the definition of 'hereditary bimodule' we see that they will hold too in $M_{2}(A)$.

$(\Leftarrow)$ Replacing by subnets, we may suppose that $c_{n}, d_{n}, c_{n} d_{n}$, and $d_{n} c_{n}$, converge in the weak* topology to say elements $u, w, p, q$, respectively. Then $c_{n} d_{n} c_{m}$ converges weak* to $u w c_{m}=c_{m}=p c_{m}$. Taking the limit over $m$ we see that $u w u=u=p u$, so $(u w)^{2}=u w=p u w$. Since $u w$ is a contraction, it is a projection. Also, $c_{n} d_{n} c_{m} d_{m}$ converges weak* to $u w c_{m} d_{m}=c_{m} d_{m}=p c_{m} d_{m}$. Taking the limit over $m$ we see that $u w p=p=p^{2}$. So $p$ is a projection too, and now we see that $p=u w$. Also $u=p u$, and similarly $w p=\lim _{n} d_{n} p=w$. It follows that $u=w^{*}$, and this is a partial isometry, and is in $\Delta\left(A^{* *}\right)$. Similarly, $q=u^{*} u$. Condition (1) implies in the limit that $p x=x=x q$ for $x \in X$, so $X \subset{ }_{p} A_{q}$. Hence $u$ is in the weak* closure of $A_{u}$. Conversely, if $y \in{ }_{p} A_{q}$ then $u^{*} y u^{*} \in A^{\perp \perp}$, and so since $X^{\perp \perp}$ is an inner ideal we see that $y=u u^{*} y u^{*} u \in X^{\perp \perp} \cap A=X$. So $X={ }_{p} A_{q}=A_{u}$. Since $\left(x d_{m} c_{m} d_{n}\right)$ converges with $n$ for fixed $m$, for $x \in X$, we have $x d_{m} c_{m} u^{*} \in A$. Taking the limit over $m$ shows that $x u^{*} \in A$. By Theorem 3.3 we see that $u$ is a *-open tripotent.

Remark. Hereditary bimodules generalize the hereditary subalgebras of [9] (called HSA's). Indeed, HSA's are just the case that $c_{n}=d_{n}$ in the characterization above. If $c_{n}=d_{n}$ in the characterization above, then $u=u^{*}$, so that $p=q$ in the proof above, and $X={ }_{p} A_{p}$ is a HSA in $A$. Conversely given a HSA with cai $\left(e_{n}\right)$, the conditions above are met with $c_{n}=d_{n}=e_{n}$.

It is clear in the definition of a hereditary bimodule $X$ at the start of this section that $D \cong E$ completely isometrically as operator algebras, and $D \cong X \cong Y$ canonically as operator algebras. In fact these isomorphisms are easy to write down in terms of the support tripotent $u$ for $X$ :

Proposition 4.3. Let $X$ be a hereditary bimodule with a support tripotent $u$. In the notation at the start of this section we have $D \cong E$ completely isometrically via the homomorphism $a \mapsto u^{*} a u$, and $X \cong Y$ completely isometrically via the linear isometry $x \mapsto u^{*} x u^{*} \stackrel{\text { def }}{=} x^{\sharp}$. Similarly, $D \cong X$ via right multiplication by $u$, and $D \cong Y$ via left multiplication by $u^{*}$.

Proof. That $D={ }_{p} A_{p} \cong{ }_{q} A_{q}=E$ via this map was done in the first paragraph of the proof of Proposition 4.1 as were the $D \cong X$ and $D \cong Y$ assertions. Similar arguments prove the other assertion: If $X=A_{u}$ for a $*$-open tripotent $u \in \Delta\left(A^{* *}\right)$, then the 'dual module' $Y=\tilde{X}$ (see the definition of a hereditary bimodule and the accompanying discussion there), is simply $v X v$ where $v=u^{*}$. Indeed as we saw in the proof of Proposition 4.1, $X u^{*}=A_{u} u^{*}=A_{u u^{*}}$, and similarly $u^{*} A_{u u^{*}}=A_{u^{*}}=$ $Y$. Thus we can define a complete isometry from $X$ onto $Y$ by $x \mapsto x^{\sharp}=v x v$. The inverse of this is of course the map $y \mapsto u y u$.

Remark. (1) One should beware though in the last result: the isomorphisms mentioned there are not unique, but depend on the particular support tripotent $u$ chosen for $X$. They are, however, determined for example by the cone $\mathbb{R}^{+} \mathfrak{S}_{A_{u}}$ in Lemma 3.4 (iii). (The nonuniqueness of the support tripotent is discussed a few paragraphs before Proposition 4.1) 
(2) Consider the bijection between *-open tripotents $v$ and the elements $x$ and $y$ in the variants of item (ii) in Theorem 2.2, described in Proposition 3.8. One may go further by saying that the $x$ and $y$ in Theorem 2.2 (ii) and its variants, are nothing but the 'square root of $a$ with respect to the tripotent' $v$. That is, for example, via the canonical isomorphisms $A_{a} \cong \overline{a A b}$ and $A_{a} \cong \overline{b A a}$ from Proposition 4.3 (but with $\left.u=v^{*}\right), x$ and $y$ both correspond to $a^{\frac{1}{2}}$. Of course $y=x^{\sharp}$ in the language of Proposition 4.3 .

We can now give several characterizations of Pedersen equivalence in terms of hereditary bimodules:

Corollary 4.4. If $a, b \in \mathfrak{S}_{A}$ then $a \sim_{A} b$ if and only if $\overline{a A b}$ is a hereditary bimodule and there exists $x \in \overline{a A b}$ such that $a=x x^{\sharp}, b=x^{\sharp} x$. Here $x^{\sharp}=u^{*} x u^{*}$ is as in Proposition 4.3, where $u$ is a *-open tripotent with $\overline{a A b}=A_{u}$.

If these hold then $\overline{a A b}$ is indeed a principal hereditary $A_{a}-A_{b}$-bimodule.

Proof. $(\Rightarrow)$ If $a \sim_{A} b$, and $u$ is as in Proposition 3.8, and $v=u^{*}$, then $A_{u}=\overline{a A b}$ by Proposition 1.6. By Proposition 4.1 this is a hereditary bimodule. The rest is clear from Remark (2) above.

$(\Leftarrow)$ Follows from Proposition 3.8 , since if $v=u^{*}$ then

$$
v a v^{*}=v x v x v v^{*}=x^{\sharp} x=b .
$$

If these hold, then as we said $\overline{a A b}$ is a hereditary bimodule. We know from [15] that $\overline{A_{a}}={ }_{p} A_{p}$ and $\overline{A_{b}}={ }_{q} A_{q}$, where $p=p_{a}, q=p_{b}$. Also, $\overline{a A b}={ }_{p} A_{q}$ by Proposition 1.6. and similarly, $\overline{b A a}={ }_{q} A_{p}$. So $\overline{a A b}$ is a principal hereditary $A_{a^{-}} A_{b^{-}}$ bimodule.

Corollary 4.5. If $a, b \in \mathfrak{S}_{A}$ and $\overline{a A b}$ is a hereditary bimodule, with nets $c_{n}, d_{n}$ as in Theorem 4.2, then $a \sim_{A} b$ if and only if we can write $a=x y, b=y x$, where $y \in \overline{b A a}$ and $x \in \overline{a A b}$, and $y c_{n}-d_{n} x \rightarrow 0$ in norm.

Proof. For the one direction, if $u$ is in the proof of Theorem 4.2, then $y u=u^{*} x$ because $y c_{n}-d_{n} x \rightarrow 0$. So $y=x^{\sharp}$ and the rest follows from Corollary 4.4. For the other direction, if $a \sim_{A} b$ then $a=x y, b=y x$ where $x=a^{1 / 2} v^{*}=v^{*} b^{1 / 2}$ and $y=v a^{1 / 2}=b^{1 / 2} v$ for open tripotents $v, v^{*} \in A^{\perp \perp}$. One can let $c_{n}=a^{1 / 2 n} v^{*}, d_{n}=$ $v a^{1 / 2 n}$, and the rest is easy.

Remark. (1) If $A=M_{n}$ then saying that $\overline{a A b}$ is a hereditary bimodule is simply saying that $\operatorname{rank}(a)=\operatorname{rank}(b)$. So we can view $\overline{a A b}$ being a hereditary bimodule as a generalization of this rank condition. If we reduce the conditions in Corollary 4.5 to say: $(a=x y, b=y x$ where $\overline{a A b}$ is a hereditary bimodule, $x \in \overline{a A b}$, and $y \in \overline{b A a})$, then one obtains a relation that is in general strictly stronger (by Corollary 5.2) than the (variant of) 'Blackadar equivalence' which we study later, and is strictly weaker in general than Pedersen equivalence.

(2) Another characterization of Pedersen equivalence: If $a, b \in \mathfrak{S}_{A}$ then $a \sim_{A} b$ iff there exists a principal hereditary $A_{a}-A_{b}$-bimodule, such that $a \oplus 0$ and $0 \oplus b$ in the linking algebra of the bimodule correspond via the map $\rho$ mentioned in the first few paragraphs of Section 4, to $c \oplus 0$ and $0 \oplus c$ for some element $c \in C$. For the one direction of this, in Corollary 4.4 we saw $\overline{a A b}$ is a principal hereditary bimodule. If $v=u^{*}$ are as in the proof of Corollary 4.4, with $v^{*} b v=a$, and if $\rho^{-1}$ is the map in the first paragraph of the proof of Proposition 4.1, then $\rho^{-1}(1 \oplus b)=1 \oplus\left(v^{*} b v\right)=$ $1 \oplus a$. For the other direction, by the proof of Proposition $4.1, \overline{a A b}=A_{u}$ for a 
*-open tripotent with $u u^{*}=p_{a}, u^{*} u=p_{b}$. If $\rho$ is as in the second paragraph of that proof, and if $\rho(c \oplus 0)=a \oplus 0$, then by hypothesis $0 \oplus b=\rho(0 \oplus c)$. However,

$$
\rho(0 \oplus c)=\rho^{* *}\left(E_{21}(c \oplus 0) E_{12}\right)=\rho^{* *}\left(E_{21}\right)(a \oplus 0) \rho^{* *}\left(E_{12}\right)=0 \oplus v a v^{*} .
$$

Thus $b=v a v^{*}$. So (iv) in Theorem 2.2 holds.

Definition 4.6. If $u$ is a $*$-open tripotent in $A^{* *}$ and $x \in \mathfrak{c}_{A_{u}}$, where the latter denotes $\mathbb{R}^{+} \mathfrak{S}_{A_{v}}$, then the $n$th roots of $x$ (with respect to the Peirce product on $\left.A_{u}\right)$, converge weak* to an open projection $s_{u}(x) \in B_{2}^{* *}(u)$. By Lemma 3.4 $s_{u}(x)$ is a $*$-open tripotent in $A^{* *}$, and $s_{u}(x) \leq u$ in the ordering on tripotents. We call $s_{u}(x)$ the $u$-support tripotent of $x$. We also call $s_{u}(x)$ a local support tripotent.

Lemma 4.7. Let $u$ be $a *$-open tripotent in $A^{* *}$ and $x \in \mathfrak{c}_{A_{u}}$. The hereditary bimodule $A_{s}$ corresponding to the local support tripotent $s=s_{u}(x)$ is a principal hereditary bimodule, indeed may be written as $\overline{a A b}$ for some $a, b \in \mathfrak{S}_{A}$ with $a \sim_{A} b$.

Proof. By [15, Corollary 2.6], working within the algebra $A_{u}$, and letting $s=s_{u}(x)$ and $v=u^{*}$, we have

$$
\overline{x v A_{u} v x}=\left\{x \in A_{u}: x=\text { svxvs }=s s^{*} x s^{*} s\right\}=\left\{x \in A: x=s s^{*} x s^{*} s\right\}=A_{s} .
$$

Also

$$
x v=x v s v=x v s s^{*} s v=x s^{*} s v=x s^{*},
$$

and similarly $v x=s^{*} x$. Let $a=x s^{*}, b=s^{*} x=s^{*} a s$. Since $s$ is $*$-open in $A^{* *}$, if $p=s s^{*}$ then right multiplication by $s^{*}$ is a completely isometric homomorphism from $A_{s}$ onto ${ }_{p} A_{p}$, which extends to a completely isometric homomorphism from $\left(A_{s}\right)^{\perp \perp}$ onto $p A^{* *} p$. It follows that $p_{a}=s s^{*}$. Similarly $p_{b}=s^{*} s$. Then $a$ and $b$ are in $\mathfrak{S}_{A}$, since for example

$$
\|1-a\|=\left\|u u^{*}-x u^{*}\right\| \leq\|u-x\| \leq 1 .
$$

Clearly $a s=x \in A$, so $a \sim_{A} b$ by Theorem 2.2 (iv)'.

Clearly, $x v A_{u} v x=a A_{u} b \subset \overline{a A b}$. To see the converse, first note that $\overline{a A}=\overline{a^{2} A}$. Indeed, squaring the power series in Lemma 1.1 shows that $a \in \overline{a^{2} A}$ and the statement is thus clear. Similarly $\overline{A b}=\overline{A b^{2}}$. So

$$
\overline{a A b}=\overline{a A A b}=\overline{a^{2} A A b^{2}} \subset \overline{a A_{u} b},
$$

the latter since $u u^{*} x=x$ and $x u^{*} u=x$. So $\overline{x u^{*} A_{u} u^{*} x}=\overline{a A b}$, and we are done.

Remark. It is easy to see that $\overline{a A b}$ in the last proof, actually equals $\overline{a A_{s_{u}(x)}}$, and also equals $\overline{A_{s_{u}(x)} b}$.

Theorem 4.8. (1) Every hereditary bimodule in $A$ is the closure of an increasing net of principal hereditary bimodules of the form $\overline{a A b}$, where $a, b \in \mathfrak{S}_{A}$ with $a \sim_{A} b$.

(2) Any *-open tripotent $u$ in $A^{* *}$ is the limit of an increasing net of local support tripotents.

Proof. (1) Viewing $A_{u}$ as an operator algebra with the Peirce product, by [15, Theorem 2.15] $A_{u}$ is the closure of an increasing net $\left(D_{t}\right)$ of 'peak principal' HSA's, each of the form $\overline{x_{t} A_{u} x_{t}}$ (with respect to the Peirce product in $A_{u}$ ), for some $x_{t} \in \mathfrak{S}_{A_{u}}$. As we saw in Lemma 4.7 we may write each $D_{t}$ as $\overline{a A b}$ for some $a, b \in \mathfrak{S}_{A}$ with $a \sim_{A} b$. 
(2) In the above, $s_{u}\left(x_{t}\right)$ forms an increasing net of open projections in $\left(A_{u}\right)^{\perp \perp}$ which converge weak ${ }^{*}$ to $u$. Hence by Lemma 3.4 they are local support tripotents, and converging weak* to $u$.

Theorem 4.9. Any separable hereditary bimodule in $A$ is a principal hereditary bimodule, that is of the form $\overline{a A b}$, where $a, b \in \mathfrak{S}_{A}$ with $a \sim_{A} b$.

Proof. If $A_{u}$ is separable, then viewing it as an operator algebra with respect to the Peirce product, we have by [15, Corollary 2.18] that $A_{u}=\overline{x A_{u} x}$ with respect to the Peirce product, for some $x \in \mathfrak{S}_{A_{u}}$. By Lemma 4.7. $A_{u}=\overline{a A b}$ for some $a, b \in \mathfrak{S}_{A}$ with $a \sim_{A} b$.

Remark. It follows as in [15. Corollary 2.18] that the cai for a separable hereditary bimodule may be chosen to be countable, and consist of mutually commuting elements in the Peirce product. We are not sure what implications this may have.

Corollary 4.10. If $A$ is a separable operator algebra, then every *-open tripotent in $A^{* *}$ is a local support tripotent $s_{u}(x)$ for some $x \in \mathfrak{S}_{A}$.

Proof. In the last proof, we have $A_{u}=A_{s_{u}(x)}$, with $s_{u}(x)$ a tripotent dominated by the $*$-open tripotent $u$. By Lemma 3.4, $u=s_{u}(x)$.

We say that a hereditary bimodule $D$ in $A$ is a hereditary subbimodule of another hereditary bimodule $D^{\prime}$ in $A$, if $D \subset D^{\prime}$, if the Peirce product of $D$ coincides with the restriction of the Peirce product of $D^{\prime}$, and $D D^{\prime} D \subset D$ in the Peirce product of $D^{\prime}$

Proposition 4.11. If a hereditary bimodule $D$ in $A$ is generated by a countable collection of principal hereditary subbimodules $D_{n}$ of $D$, (so in the Peirce product on $D$ there is no proper $H S A$ containing all the $D_{n}$ ), then $D$ is a principal hereditary bimodule in $A$.

Proof. This follows from [15. Theorem 2.16 (2)] in the same way that the last several results followed from matching results in [15].

For interests sake we give a generalization of Theorem 4.9 to a slightly more general class of inner ideals in $A$. Similarly to our first definition of a hereditary bimodule at the start of this section, suppose that we have subalgebras $D, E$ of $A$, and subspaces $X, Y$ of $A$, such that $(D, E, X, Y)$ is a strong Morita equivalence context in the sense of 11, and the linking algebra of this context, namely the subalgebra $\mathcal{L}$ of $M_{2}(A)$ with rows $C, X$ and $Y, D$, is a HSA in $M_{2}(A)$. Then we say that $X$ is an inner equivalence bimodule, or an inner equivalence $D$-E-bimodule. Note that again $D=X Y, E=Y X, X=D A E, Y=E A D$, and $D$ and $E$ are HSA's and $X$ and $Y$ are inner ideals in $A$. These follow by the same reasons as before. A principal inner equivalence bimodule, or a principal inner equivalence $A_{a^{-}}$ $A_{b}$-bimodule, is the case when the context is $\left(A_{a}, A_{b}, \overline{a A b}, \overline{b A a}\right)$ for some $a, b \in \mathfrak{S}_{A}$. Remark (1) before Theorem2.2 essentially says that if $a \sim_{r} b$ then $\overline{a A b}$ is a principal inner equivalence $A_{a}-A_{b}$-bimodule.

Theorem 4.12. Any inner equivalence bimodule in A which is also separable, is a principal inner equivalence bimodule.

Proof. Let $(D, E, X, Y)$ is as above, and suppose that the inner equivalence bimodule $X$ is separable. Then the ternary envelope $Z=\mathcal{T}(X)$ is separable (see e.g. 
8, 10, for a discussion of the ternary envelope, sometimes called the triple envelope). By [8, Lemma 10.2], the TRO $Z$ is isomorphic to a canonical $C^{*}$-algebraic Morita equivalence bimodule $Z$ containing $X$. By facts in e.g. p. 407 of [7], $Z^{*}$ contains $Y, Z Z^{*}$ contains $D$, and $Z^{*} Z$ contains $E$. So $D, E, Y$ are each separable. By [15, Theorem 2.16 (1)], $D=A_{a}, E=A_{b}$ for some $a, b \in \mathfrak{S}_{A}$. Thus $X=D A E \subset \overline{a A b}$, and conversely $\overline{a A b} \subset D A E=X$ since $a \in D, b \in E$. So $X=\overline{a A b}$, and similarly $Y=\overline{b A a}$.

\section{BlackAdAR EQUivalence}

For $a, b \in \mathfrak{S}_{A}$ we define $a \cong b$ if $s(a)=s(b)$. By the theory in [9, Section 2] this is equivalent to $\overline{a A}=\overline{b A}$, and also equivalent to $\overline{a A a}=\overline{b A b}$.

If $a, b \in \mathfrak{c}_{A}$ we define $a \sim_{s} b$ if there exist $a^{\prime}, b^{\prime} \in \mathfrak{S}_{A}$, with $a \cong a^{\prime}, a^{\prime} \sim_{A} b^{\prime}$, and $b^{\prime} \cong b$. It will follow from the next result that this is an equivalence relation. We say that $a$ and $b$ are Blackadar equivalent in $A$ if $a \sim_{s} b$.

Theorem 5.1. (Cf. 29], 31, Proposition 4.3].) For $a, b \in \mathfrak{c}_{A} T F A E$ :

(i) $a \sim_{s} b$.

(ii) $\overline{a A} \cong \overline{b A}$ completely isometrically via a right $A$-module map.

(iii) $p_{a} \sim_{P Z, A} p_{b}$.

(iv) There exists $b^{\prime} \in \mathfrak{c}_{A}$, with $a \sim_{A} b^{\prime}$ and $b^{\prime} \cong b$.

(v) There exists $a^{\prime} \in \mathfrak{c}_{A}$, with $a \cong a^{\prime} \sim_{A} b$.

(vi) $\overline{a A b}$ is a principal hereditary $A_{a}-A_{b}$-bimodule.

Proof. We may assume that $a, b \in \mathfrak{S}_{A}$.

(iv) $\Rightarrow$ (vi) There exists $f \in \mathfrak{c}_{A}$ with $a \sim_{A} f$ and $f \cong b$. By Proposition 4.4 $\overline{a A f}$ is a principal hereditary bimodule, but $\overline{f A f}=\overline{b A b}$.

(vi) $\Rightarrow$ (iii) By Proposition 4.1 we have $X=\overline{a A b}=A_{u}$ for a $*$-open tripotent $u \in A^{\perp \perp}$. Note that $p_{a} x=x$ for all $x \in X$, so that $p_{a} u=u$. Hence $p_{a} u u^{*}=u u^{*}$, so $u u^{*} \leq p_{a}$. Similarly, $u^{*} u \leq p_{b}$. However since $a \in A_{a}=X Y$, we have $u u^{*} a=a$, so that $u u^{*} \leq p_{a}$. Hence $u u^{*}=p_{a}$ and similarly $u^{*} u=p_{b}$. Of course $u a \in A$ by definition of a $*$-open tripotent.

(iv) $\Rightarrow$ (i) Trivial.

(ii) $\Rightarrow$ (iv) If $\Phi: \overline{a A} \rightarrow \overline{b A}$ is a surjective completely isometric right $A$-module map, then by [7. Corollary 3.7] there exists a surjective completely isometric left $A$-module map $\Psi: \overline{A a} \cong \overline{A b}$ such that $\Psi(y) \Phi(x)=y x$ for all $x \in a A, y \in A a$.

We obtain using [7, Theorem 6.8], a $C^{*}$-module isomorphism

$$
B \otimes_{h A} \overline{A a} \rightarrow B \otimes_{h A} \overline{A b}
$$

Now the multiplication map $m: B \otimes_{h A} \overline{A a} \rightarrow \overline{B a}$ has an asymptotic contractive left inverse $\theta_{n}: z \mapsto z \otimes a^{\frac{1}{n}}$. Indeed

$$
\theta_{n}(m(b \otimes x))=b x \otimes a^{\frac{1}{n}}=b \otimes x a^{\frac{1}{n}} \rightarrow b \otimes x .
$$

for all $b \in B, x \in \overline{A a}$. So $m$ is isometric, and so $\Psi$ extends to a $C^{*}$-module map $\overline{B a} \rightarrow \overline{B b}$ which is unitary onto its range. It follows from e.g. [10, Corollary 8.1.8] that $y^{*} y=\left(a^{\frac{1}{2}}\right)^{*} a^{\frac{1}{2}}$, where $y=\Phi\left(a^{\frac{1}{2}}\right)$. Similarly, $x x^{*}=a^{\frac{1}{2}}\left(a^{\frac{1}{2}}\right)^{*}$ if $x=\Psi\left(a^{\frac{1}{2}}\right)$, and $\left.x y=\Psi\left(a^{\frac{1}{2}}\right) \Phi\left(a^{\frac{1}{2}}\right)=a^{\frac{1}{2}} a^{\frac{1}{2}}\right)=a$. By Theorem [2.7 $b^{\prime}=y x \in \mathfrak{c}_{A}$ and $a \sim_{A} b^{\prime}$. Finally, $\overline{b^{\prime} A}=\overline{y x A} \subset \overline{y A}$. On the other hand, $y=\lim _{n} y a^{\frac{1}{n}} \in y \overline{a A} \subset \overline{y x A}$, since $a=x y$. So

$$
\overline{b^{\prime} A}=\overline{y A}=\overline{\Phi\left(a^{\frac{1}{2}}\right) A}=\Phi\left(\overline{a^{\frac{1}{2}} A}\right)=\overline{b A} .
$$


Thus $a \sim_{A} b^{\prime} \cong b$.

(i) $\Rightarrow$ (iii) If $a \sim_{A} b$ and $v$ is as in the proof of Theorem 2.2 above, then $v, v^{*} \in A^{\perp \perp}$ as we said in Theorem 2.2. Thus $p_{a} \sim_{P Z, A} p_{b}$ via $v$ (for example, $v^{*} b=v^{*} d d=x d \in A$ so $v^{*} B_{q}=v^{*} \overline{b B b} \subset B$ ). Since $p_{a}=p_{a^{\prime}}$ if $a \cong a^{\prime}$ (iii) is now clear.

(iii) $\Rightarrow$ (ii) If $p_{a} \sim_{\mathrm{PZ}, \mathrm{A}} p_{b}$ via a partial isometry $v$, let $\Phi(x)=v x$ and $\Psi(x)=$ $x v^{*}$. These are the desired completely isometric module maps in (ii) (note that $\Phi(a A)=v a A \subset v A_{p} A \in A \cap q B^{* *}=\overline{b A}$, and similarly $\left.\Psi(b A) \subset a A\right)$.

The proof of the equivalence with $(\mathrm{v})$ is similar to the proof of the equivalence with (iv).

Remark. For algebras without any nontrivial r-ideals all elements of $\mathfrak{c}_{A}$ are Blackadar equivalent to each other of course. Such algebras are discussed in [15.

Corollary 5.2. If $a, b \in \mathfrak{c}_{A}$, and $X=\overline{a A b}$ and $Y=\overline{b A a}$ then TFAE:

(i) $p_{a} \sim_{P Z, A} p_{b}$.

(ii) There are nets of contractions $c_{n} \in X$, and $d_{n} \in A$ satisfying (2) of Theorem 4.2, and also $c_{n} d_{n} a \rightarrow a$ and $b d_{n} c_{n} \rightarrow b$ in norm.

(iii) $X$ is a hereditary bimodule and also $a \in X Y, b \in Y X$.

Proof. (ii) $\Rightarrow$ (i) These conditions guarantee by Theorem 4.2 that $X$ is a hereditary bimodule, and $X=A_{u}$ for a $*$-open tripotent $u \in A^{\perp \perp}$. As in the proof of Theorem [5.1. $u u^{*} \leq p_{a}$ and $u^{*} u \leq p_{b}$. However, the last condition in (ii), together with a part of the proof of Theorem 4.2, guarantees that $u u^{*} a=a$ and $b u^{*} u=b$. So $u u^{*}=p_{a}$ and $u^{*} u=p_{b}$.

(i) $\Rightarrow$ (iii) Follows from Theorem 5.1,

(iii) $\Rightarrow$ (ii) Follows from Theorem 4.2

We now give some other conditions that imply Blackadar equivalence in a $C^{*}$ algebra.

Theorem 5.3. Suppose that $a, b \in \mathfrak{c}_{A}$ for an operator algebra $A$. If $a=x y$ and $b=y x$ for some $x, y \in A$, then $p_{a} \sim_{P Z} p_{b}$ in $B^{* *}$.

Proof. Without loss of generality, by dividing by a suitable scalar, we may assume that $a, b \in \mathfrak{S}_{A}$. Under these conditions $a x=x y x=x b$, and hence $a^{\frac{1}{n}} x=x b^{\frac{1}{n}}$ for all $n \in \mathbb{N}$ (as in Remark (2) before Theorem 2.2). In the limit, $p x=x q$ and $x^{*} p=q x^{*}$, where $p=p_{a}$ and $q=p_{b}$. It follows that $p x x^{*} x=x x^{*} x q$, and similarly for fivefold and sevenfold products of this type, etc. Thus $\operatorname{pr}(x)=r(x) q$, where $r(x)$ is the range tripotent of $x$ in $Z^{* *}$, because $r(x)$ is a weak* limit of linear combinations of such 'odd products' (see e.g. the proof of [12, Lemma 3.3]). Since $p$ is a limit of functions of $x y$, clearly $r(x) r(x)^{*} p=p$. Thus $w=p r(x)$ is a tripotent and it is easy to see that $w w^{*}=p$ and $w^{*} w=q$. Note that $w^{*} a=r(x)^{*} x y=|x| y \in B$. So $w^{*}{ }_{p} B_{p}=w^{*} \overline{a B a} \subset B$. By [34, Lemma 1.3] we have $p_{a} \sim_{P Z} p_{b}$.

It is not necessarily true that if $a=x y$ and $b=y x$ for some $x, y \in A$, then $a$ and $b$ are Blackadar equivalent in $A$, if $A$ is nonselfadjoint. For a counterexample, let $R$ be an invertible operator on a Hilbert space $H$, and let $A$ be the span in $M_{2}(B(H))$ of $a=I_{H} \oplus 0, b=0 \oplus I_{H}, x=E_{12} \otimes R, y=E_{21} \otimes R^{-1}$. Then $a=x y$ and $b=y x$, but $a$ and $b$ are not Blackadar equivalent in $A$ if $R$ is chosen appropriately.

This counterexample also shows that the conditions considered in (2) of the next result also do not characterize Blackadar equivalence in $A$ if $A$ is nonselfadjoint. 
Proposition 5.4. Let $A$ be an approximately unital operator algebra, suppose that $a, b \in \mathfrak{c}_{A}$, and let $B$ be $a C^{*}$-algebra containing $A$ as usual. Then

(1) Suppose that for some $x \in A$ we have $\overline{a A}=\overline{x A}$, and $\overline{A b}=\overline{A x}$. Then $a$ and $b$ are Blackadar equivalent in $B$.

(2) If $a$ and $b$ are Blackadar equivalent in $A$ then there exists an $x \in A$ such that $\overline{a A}=\overline{x A}$, and $\overline{A b}=\overline{A x}$.

(3) $a$ and $b$ are Blackadar equivalent in $B$ if and only if there exists an $x \in B$ such that $\overline{a A}=\overline{x A}$, and $\overline{A b}=\overline{A x}$.

Proof. (1) Let $p=p_{a}, q=p_{b}$. If these conditions hold then $x \in \overline{a A} \cap \overline{A b}$, so $p x=p x q=x q$. Since $a \in \overline{x A}$ we have $r(x) r(x)^{*} p=p$, and the proof of (1) then follows as in the previous theorem.

(2) These follow for example by the same reasoning used in the proof of Theorem 5.1 to show that $\overline{y A}=\overline{b^{\prime} A}=\overline{b A}$.

(3) This is now obvious.

Remark. (1) If $A$ is a $C^{*}$-algebra then the last result gives an alternative characterization of Peligrad-Zsidó/Blackadar equivalence. One may ask what is the least restrictive extra condition we can add to these to characterize our variant of Blackadar equivalence in a more general operator algebra. For example, one such extra condition is that $\overline{a A b}$ is a hereditary bimodule (see Corollary 5.2 (iii)).

(2) Another relation equivalent to the one considered in the last result, is that $\overline{A_{a} x}=\overline{x A_{b}}$ for some $x \in A$, and $a \in \overline{x A}$ and $b \in \overline{B x}$. This also characterizes Peligrad-Zsidó/Blackadar equivalence in a $C^{*}$-algebra.

In the remainder of this section, we turn to the topic of subequivalence.

For any $a, b \in \mathfrak{c}_{A}$ we have as in 4.4 in [31, that $a \in A_{b}$ iff $A_{a} \subset A_{b}$ iff $\overline{a A} \subset \overline{b A}$ iff $p_{a} \leq p_{b}$. Also $\left(a \in A_{b}\right.$ and $\left.b \in A_{a}\right)$ iff $a \cong b$ iff $\overline{a A}=\overline{b A}$ iff $p_{a}=p_{b}$.

Lemma 5.5. Suppose that $a \in \mathfrak{c}_{A}$, and that $\Phi, \Psi$ are a pair of completely contractive $A$-module maps from $\overline{a A}$ and $\overline{A a}$ into $A$, such that $\Psi(x) \Phi(y)=x y$ for all $x \in \overline{A a}, y \in \overline{a A}$. Then $\Phi, \Psi$ are completely isometric, and there exists $b \in \mathfrak{c}_{A}$ with $a \sim_{A} b$ and $\operatorname{Ran}(\Phi)=\overline{b A}$ and $\operatorname{Ran}(\Psi)=\overline{A b}$.

Proof. Let $F, E$ be the ranges of these two maps, which are respectively a right and a left ideal in $A$. By applying the $\otimes_{h A}$ tensor product with $B$ as in the proof that (ii) $\Rightarrow$ (iv) in Theorem 5.1 above, we may extend $\Phi, \Psi$ to contractive $B$-module maps from $\overline{a B}$ and $\overline{B a}$ into $B$. If $\Phi(c)=y, \Psi(c)=x$, where $c=a^{\frac{1}{2}}$, then $y^{*} y \leq c^{*} c$ by e.g. 8.1.5 in [10], and similarly $x x^{*} \leq c c^{*}$. By basic operator theory, $y=S c, x=c R$ for contractions $S, R$. By the proof that (ii) $)^{\prime \prime \prime}$ implies (ii) ${ }^{\prime}$ in Theorem 2.2, we actually have $|y|=|c|,\left|x^{*}\right|=\left|c^{*}\right|$. Thus $\Phi(c z)^{*} \Phi(c w)=z^{*} y^{*} y w=(c z)^{*}(c w)$, for all $z, w \in A$, which forces $\Phi$ to be a complete isometry. Similarly $\Psi$ is a complete isometry. Setting $b=y x$, then $b \in \mathfrak{c}_{A}$ by Theorem 2.7. and $a \sim_{A} b$. Also, $b A=y x A \subset \overline{y A}$, and conversely, $y a^{\frac{1}{n}} \in \overline{y A} \subset \overline{b x A} \subset \overline{b A}$, so $y \in \overline{b A}$ and $\overline{y A} \subset \overline{b A}$. Thus $\overline{y A}=\overline{b A}$. Hence $F=\Phi(\overline{a A})=\Phi(\overline{c A})=\overline{y A}=\overline{b A}$. Similarly, $E=\overline{A b}$.

If $a, b \in \mathfrak{c}_{A}$, we define $a \precsim_{s} b$ if there exists $b^{\prime} \in A_{b}$ such that $a \sim_{s} b^{\prime}$. This is clearly equivalent to: there exists $b^{\prime} \in A_{b}$ such that $a \sim_{A} b^{\prime}$. We will call this Blackadar comparison in $A$. If $p, q$ are open projections in $A^{* *}$ we say that $p \precsim_{P Z, A} q$ if there is an open projection $q^{\prime} \leq q$ in $A^{* *}$ with $p \sim_{P Z, A} q^{\prime}$. We will call this Peligrad-Zsidó subequivalence in $A^{* *}$. 
The following is the version of [31, Proposition 4.6] in our setting (see also [29]):

Proposition 5.6. If $a, b \in \mathfrak{c}_{A}, T F A E$ :

(i) $a \precsim_{s} b$.

(ii) $p_{a} \precsim P Z, A p_{b}$.

(iii) There exist a pair of completely contractive A-module maps $\Phi: \overline{a A} \rightarrow \overline{b A}$ and $\Psi: \overline{A a} \rightarrow \overline{A b}$, such that $\Psi(x) \Phi(y)=x y$ for all $x \in \overline{A a}, y \in \overline{a A}$.

Proof. (iii) $\Rightarrow$ (i) By Lemma 5.5. (iii) implies that there exist $x, y \in \operatorname{Ball}(A)$ with $a=x y, b^{\prime}=y x \in \mathfrak{c}_{A}$. In our case, $b^{\prime}=y x \in A_{b}$ too.

(i) $\Rightarrow$ (ii) By Theorem [5.1, $p_{a} \sim_{P Z, A} p_{b^{\prime}}$, and clearly $p_{b^{\prime}} \leq p_{b}$.

(ii) $\Rightarrow$ (iii) If we have an open projection $q^{\prime} \leq p_{b}$ in $A^{* *}$ with $p_{a} \sim_{P Z, A} q^{\prime}$ via a tripotent $v$, then by Proposition 3.9 we have $q^{\prime}=v v^{*}=p_{b^{\prime}}$ where $b^{\prime}=v a v^{*}$. Left multiplication by $v$ is a completely isometric module map from $\overline{a A}$ into $A$. Note that $p_{b} v a=p_{b} v v^{*} v a=p_{b} p_{b^{\prime}} v a=p_{b^{\prime}} v a=v a$. So the map maps into $p_{b} A=\overline{b A}$. Similarly, right multiplication by $v^{*}$ is a completely isometric module map from $\overline{A a}$ into $\overline{A b}$. The rest is clear.

As in [31, Proposition 4.6], these give $x, y \in \operatorname{Ball}(A)$ such that $\overline{a A}=\overline{x y A}$ and $\overline{y x A} \subset \overline{b A}$, and so on. Here $y x \in \mathfrak{c}_{A}$.

Remark. In contrast to Theorem 5.1(ii), one needs the statement about $\Psi$ in (iii) of Proposition 5.6. It is not automatic, as one may see by considering for example multiplication by $z$ on the disk algebra.

\section{Appendix: Some Related Results for TROs}

We include the following results about TROs for the reason that, as we claimed in Remark (2) at the start of Section 3, many of our results generalize easily to the case that the operator algebra $A$ is replaced by a strong Morita equivalence bimodule in the sense of [11. The results below show how this can proceed in the case of TROs.

If $v$ is a tripotent in the second dual of a TRO $Z$ such that $p=v^{*} v$ and $q=v v^{*}$ are open projections with respect to $Z^{*} Z$ and $Z Z^{*}$ respectively, and if $v\left(Z Z^{*}\right)_{p} \subset Z, v^{*}\left(Z Z^{*}\right)_{q} \subset Z^{*}$, then we say that $v$ implements a TRO PeligradZsidó equivalence of open projections. This is of course equivalent to $v$, thought of as in the second dual of the linking $C^{*}$-algebra of $Z$, implementing a Peligrad-Zsidó equivalence in the original sense between $0 \oplus p$ and $q \oplus 0$. So again we need not say $v^{*}\left(Z Z^{*}\right)_{q} \subset Z^{*}$ in this definition. The following proof shows that the two 'inclusion conditions' are equivalent to: $v^{*}\left({ }_{q} Z_{p}\right) \subset Z^{*} Z$ and $v\left({ }_{p} Z_{q}^{*}\right) \subset Z Z^{*}$.

The following (which we should have seen in [12]), is the appropriate variant of the equivalence (i) $\Leftrightarrow(\mathrm{vi})$ in [16]:

Proposition 6.1. If $v$ is a tripotent in the second dual of a TRO Z, then $v$ implements a TRO Peligrad-Zsidó equivalence of open projections iff $v$ is an open tripotent in the sense of [12].

Proof. $(\Rightarrow)$ Under these conditions, note that

$$
D=Z(v)=Z_{2}^{* *}(v) \cap Z=\{b \in Z: q b p=b\}
$$


is an inner ideal in $Z$. Here $p=v^{*} v, q=v v^{*}$. It is also a $C^{*}$-subalgebra of $Z^{* *}(v)$. Indeed

$$
v^{*} D=v^{*} D D^{*} D \subset Z^{*} D \subset Z^{*} Z
$$

and

$$
v D^{*}=v D^{*} D D^{*} \subset Z D^{*} \subset Z Z^{*},
$$

hence $D v^{*} D \subset Z \cap Z^{* *}(v)=D$, and $v D^{*} v=v D^{*} D D^{*} v \subset Z Z^{*} D Z^{*} Z \subset Z$, so $D v^{*} D \subset Z \cap Z^{* *}(v)=D$. If $B=Z^{*} Z$ then $B_{p}=\{b \in B: p b p=b\}$ is a HSA with support projection $p$, and $v^{*} D=B_{p}$ (clearly $v^{*} D \subset B_{p}$ and conversely if $b \in B_{p}$ then $v b \subset Z \cap Z_{2}^{* *}(v)=D$, so $\left.b \in v^{*} D\right)$. So left multiplication $L_{v^{*}}$ by $v^{*}$ is a linear complete isometry from $D$ onto $B_{p}$, and is a ternary isomorphism, and indeed it is a $*$-isomorphism with respect to the $v$-product on $D$. It follows that $D^{*} D=B_{p}$, and similarly $D D^{*}=\left(Z Z^{*}\right)_{q}$, although these are clear directly: $D^{*} D=D^{*} v v^{*} D=B_{p}^{*} B_{p}=B_{p}$.

Suppose that $x_{t}$ is a positive cai in the $C^{*}$-algebra $D$ with the $v$-product, with weak* limit $w$. This will be an open tripotent in the sense of [12] dominated by $v$. Then $v^{*} x_{t}$ is a positive cai in $B_{p}$, so that in the limit we have $v^{*} w=p$. Since $w p=w$ and $v p=v$, it follows from a basic operator theory fact that $v=w$. So $v$ is open in the sense of 12 .

$(\Leftarrow)$ If $v$ is open, then by the remark after Corollary 2.11 in [12, $p$ and $q$ are open. If $x \in Z(v)$ then $v^{*} v x^{*} x=x^{*} x \subset Z^{*} Z$. Since $v x^{*} x$ is the generic positive element in the $C^{*}$-algebra $Z(v)$, it follows that $v^{*} Z(v) \subset Z^{*} Z$. Similarly, $Z(v) v^{*} \subset Z Z^{*}$. We note that the HSA $Z(v) Z(v)^{*}$ of $Z Z^{*}$ has support projection $q$, by the first part of the proof, and similarly $Z(v)^{*} Z(v)$ has support projection $p$. So $v^{*}\left(Z Z^{*}\right)_{q}=v^{*} Z(v) Z(v)^{*} \subset Z^{*} Z Z(v)^{*} \subset Z^{*}$, and similarly $v\left(Z Z^{*}\right)_{p} \subset Z$.

Proposition 6.2. Let $Z$ be a TRO, set $A=Z Z^{*}, B=Z^{*} Z$, and let $v$ be an open tripotent in $Z^{* *}$, and suppose that $p=v^{*} v$ is the support projection for some $b \in$ $\mathfrak{S}_{B}$. Then $q=v v^{*}$ is the support projection for some $a \in \mathfrak{S}_{A}$, and in this case there exist $x, y \in \operatorname{Ball}(Z)$ with $x \in{ }_{q} Z_{p}, y \in{ }_{p} Z_{q}$, and $x y=a, y x=b$. Moreover, $\overline{a A a}$ and $\overline{b B b}$ are $*$-isomorphic $C^{*}$-algebras. They are also strongly Morita equivalent via the equivalence bimodule $\overline{a Z b}=\overline{b Z * a}^{*}$, and this bimodule is ternary isomorphic to $\overline{b B b}$.

Proof. As in Proposition 3.3 of [31, let $a=v b v^{*}$. Note that $a=v b^{\frac{1}{2}} b^{\frac{1}{2}} v^{*} \in$ $v B_{p}\left(v B_{p}\right)^{*} \subset A_{q}$. Also, $a \in \mathfrak{S}_{A}$ by Lemma 1.3 and $\left(v b v^{*}\right)^{\frac{1}{n}}=v b^{\frac{1}{n}} v^{*}$ by Lemma 1.4. Then

$$
p_{a}=\lim _{n}\left(v b v^{*}\right)^{\frac{1}{n}}=\lim _{n} v b^{\frac{1}{n}} v^{*}=v p_{b} v^{*}=q,
$$

where these are weak ${ }^{*}$ limits. Hence $q$ is the support projection of $a$. If $x=v b^{\frac{1}{2}}$ and $y=b^{\frac{1}{2}} v^{*}$, then $x y=a, y x=b$ since $b^{\frac{1}{2}} \in B_{p}$.

Continuing as in [15], $\overline{b B b}=B_{p}$ since $p=s(b)$, and similarly $\overline{a A a}=A_{q}$. These are $C^{*}$-algebras in this case. Also $a Z b \subset{ }_{q} Z_{p}$, and, conversely, we have ${ }_{q} Z_{p}=$ $A_{q} Z_{p} B_{p} \subset \overline{a Z b}$. So $\overline{a Z b}={ }_{q} Z_{p}$, and similarly $\overline{b Z^{*} a}={ }_{p} Z_{q}^{*}$. So we have a $C^{*}$ algebraic Morita equivalence between $\overline{a A a}$ and $\overline{b B b}$, implemented by the equivalence bimodule $\overline{a Z b}={\overline{b Z^{*} a}}^{*}$.

The last assertions follow from the first paragraph of Proposition 6.1. Note that $z \mapsto v z v^{*}$ is a $*$-isomorphism from $A_{p}$ onto $B_{q}$, with inverse the map $z \mapsto v^{*} z v$. 
Proposition 6.3. Any separable inner ideal $D$ in a TRO $Z$ is of the form $\overline{a Z b}$ for $a \in\left(Z Z^{*}\right)_{+}, b \in\left(Z^{*} Z\right)_{+}$. If $D$ is also ternary isomorphic to a $C^{*}$-algebra then this can be done with $p_{a} \sim_{P Z} p_{b}$.

Proof. Let $A=Z Z^{*}, B=Z^{*} Z$. If $a \in A_{+}, b \in B_{+}$then $\overline{a Z b}$ is an inner ideal. Conversely, if $D$ is an inner ideal in $Z$, then $D D^{*}$ is a HSA in $A$, which is separable if $D$ is separable. Hence $D D^{*}=\overline{a A a}$ for some $a \in A_{+}$. Similarly, $D^{*} D=\overline{b B b}$ for some $b \in B_{+}$. Thus $D=D D^{*} D D^{*} D=\overline{a Z b}$ (since $a Z b \subset D\left(D^{*} Z D^{*}\right) D \subset D$ because $D$ is an inner ideal). If $D$ is also ternary isomorphic to a $C^{*}$-algebra $C$ then the identity in $C^{* *}$ corresponds to an open tripotent $u \in D^{* *}$ by [12, Proposition $3.5]$, and it is easy to argue that $u u^{*}\left(\right.$ resp. $u^{*} u$ ) is the support projection of $D D^{*}$ (resp. $\left.D^{*} D\right)$. So $u u^{*}\left(\right.$ resp. $u^{*} u$ ) is the support projection of $a$ (resp. $b$ ).

Acknowledgements: The second author was supported by Denison University.

\section{REFERENCES}

[1] C. A. Akemann, The general Stone-Weierstrass problem, J. Funct. Anal. 4 (1969), 277-294.

[2] C. A. Akemann, Left ideal structure of $C^{*}$-algebras, J. Funct. Anal. 6 (1970), 305-317.

[3] C. A. Akemann and G. K. Pedersen, Facial structure in operator algebra theory, Proc. London Math. Soc. 64 (1992), 418-448.

[4] P. Ara, F. Perera, and A. S. Toms, K-Theory for operator algebras. Classification of $C^{*}$ algebras, in: Aspects of operator algebras and applications, 171, Contemp. Math., 534, Amer. Math. Soc., Providence, RI, 2011.

[5] W. B. Arveson, Subalgebras of $C^{*}$-algebras, Acta Math. 123(1969), 141-224.

[6] B. Blackadar, Operator algebras. Theory of $C^{*}$-algebras and von Neumann algebras, Encyclopaedia of Mathematical Sciences Vol. 122, Operator Algebras and Non-commutative Geometry, III, Springer-Verlag, Berlin, 2006.

[7] D. P. Blecher, A generalization of Hilbert modules, J. Funct. Anal. 136 (1996), 365-421.

[8] D. P. Blecher, Multipliers, $C^{*}$-modules, and algebraic structure in spaces of Hilbert space operators, in: Operator algebras, quantization, and noncommutative geometry: A centennial celebration honoring J. von Neumann and M. H. Stone, Contemp. Math., 365, Amer. Math. Soc., 2004.

[9] D. P. Blecher, D. M. Hay, and M. Neal, Hereditary subalgebras of operator algebras, J. Operator Theory 59 (2008), 333-357.

[10] D. P. Blecher and C. Le Merdy, Operator algebras and their modules-an operator space approach, Oxford Univ. Press, Oxford (2004).

[11] D. P. Blecher, P. S. Muhly, and V. I. Paulsen, Categories of operator modules (Morita equivalence and projective modules), Mem. Amer. Math. Soc. 681 (2000).

$[12]$ D. P. Blecher and M. Neal, Open partial isometries and positivity in operator spaces, Studia Math. 182 (2007), 227-262.

[13] D. P. Blecher and M. Neal, Open projections in operator algebras II: Compact projections, Preprint 2010.

[14] D. P. Blecher, K. Kirkpatrick, M. Neal, and W.Werner, Ordered involutive operator spaces, Positivity 11 (2007), 497-510.

[15] D. P. Blecher and C. J. Read, Operator algebras with contractive approximate identities, J. Funct. Anal. 261 (2011), 188-217.

[16] D. P. Blecher and W.Werner, Ordered $C^{*}$-modules, Proc. London Math. Soc. 92 (2006), 682-712.

[17] K. T. Coward, G. A. Elliott, C. Ivanescu, The Cuntz semigroup as an invariant for Calgebras, J. Reine Angew. Math. 623 (2008), 161193.

[18] C. M. Edwards, F. J. Fernandez-Polo, C. S. Hoskin, and A. M. Peralta, On the facial structure of the unit ball in a JB*-triple, J. Reine. Angew. Math. 641 (2010), 123-144.

[19] C. M. Edwards, K. McCrimmon, and G. T. Rüttimann, The Range of a Structural Projection, J. Funct. Anal. 139 (1996), 196-224.

[20] C. M. Edwards and G. T. Rüttimann, Inner ideals in $C^{*}$-algebras, Math. Ann. 290 (1991), 621-628. 
[21] C. M. Edwards and G. T. Rüttimann, Exposed faces of the unit ball in a JBW*-triple, Math. Scand. 82 (1998), no. 2, 287-304.

[22] F. J. Fernandez-Polo and A. M. Peralta, Non-commutative generalisations of Urysohn's lemma and hereditary inner ideals, J. Funct. Anal. 259 (2010), 343-358.

[23] F. J. Fernandez-Polo and A. M. Peralta, On the facial structure of the unit ball of the dual space of a JB*-triple, Math. Ann. 348 (2010), 1019-1032.

[24] T. W. Gamelin, Uniform Algebras, Second edition, Chelsea, New York, 1984.

[25] D. M. Hay, Closed projections and peak interpolation for operator algebras, Integral Equations Operator Theory 57 (2007), 491-512.

[26] D. M. Hay, Multipliers and hereditary subalgebras of operator algebras, Studia Math. 205 (2011), 31-40.

[27] C. R. Johnson and E. A. Schreiner, The relationship between $A B$ and BA, Amer. Math. Monthly 103 (1996), 578-582.

[28] C-K. Li, L. Rodman, and I. M. Spitkovsky, On numerical ranges and roots, J. Math. Anal. Appl. 282 (2003), 329-340.

[29] H. Lin, Cuntz semigroups of $C^{*}$-algebras of stable rank one and projective Hilbert modules, Preprint (2010), arXiv:1001.4558

[30] M. Mbekhta, Partial isometries and generalized inverses, Acta Sci. Math. (Szeged) 70 (2004), 767-781.

[31] E. Ortega, M. Rørdam, and H. Thiel, The Cuntz semigroup and comparison of open projections, J. Funct. Anal. 260 (2011), 3474-3493.

[32] G. K. Pedersen, $C^{*}$-algebras and their automorphism groups, Academic Press, London (1979).

[33] G. K. Pedersen, Factorization in $C^{*}$-algebras, Exposition. Math. 16 (1998), 145-156.

[34] C. Peligrad and L. Zsidó, Open projections of $C^{*}$-algebras: comparison and regularity, Operator theoretical methods (Timisoara, 1998), 285-300, Theta Found., Bucharest, 2000.

[35] C. J. Read, On the quest for positivity in operator algebras, J. Math. Anal. Appl. 381 (2011), 202-214

Department of Mathematics, University of Houston, Houston, TX 77204-3008

E-mail address, David P. Blecher: dblecher@math.uh.edu

Department of Mathematics, Denison University, Granville, OH 43023

E-mail address: nealm@denison.edu 